\title{
Molecular $K$-shell photoionization cross sections in the relaxed-core Hartree-Fock approximation
}

\author{
J. Schirmer, ${ }^{*}$ M. Braunstein, and V. McKoy \\ Arthur Amos Noyes Laboratory of Chemical Physics, California Institute of Technology, Pasadena, California 91125
}

(Received 30 May 1989)

\begin{abstract}
The relaxed-core Hartree-Fock (RCHF) approach to the calculation of $K$-shell photoionization cross sections is analyzed and applied to $K$-shell single-hole ionization in CO. A direct method based on the Schwinger variational principle and single-center-expansion techniques is used to generate the continuum orbitals associated with the motion of the photoelectron in the direct and exchange potential of the relaxed ion. A method is presented for evaluating the $N$-electron transition moment, a step that has posed a considerable computational obstacle due to the lack of orthogonality between the frozen and relaxed orbitals in the initial and final $N$-electron states, respectively. Besides being very practical and efficient, this formulation establishes the distinction between the "direct" and "conjugate" part of the transition moment, introducing bound-free dipole and overlap integrals, respectively. Whereas for large photoelectron energies the conjugate terms can be neglected, they become important near threshold, contributing, for example, up to $30 \%$ to the $1 \mathrm{~s}$ cross sections in CO. An analysis by means of low-order perturbation theory shows that the RCHF model correctly describes the effect of ionic relaxation, that is, essentially the screening of the $1 s$ hole by the valence electrons. As a consequence the $\sigma^{*}$ shape resonance is substantially shifted to higher energy and broadened compared with the frozen-core Hartree-Fock picture where the more attractive unscreened $1 s$-hole potentials are used. The remaining discrepancies with the experimental results are attributed to the neglect of target polarization in the RCHF model.
\end{abstract}

\section{INTRODUCTION}

Photoionization of atoms or molecules with sufficiently energetic photons generates highly excited ionic states characterized by a $K$-shell electron vacancy. ${ }^{1,2}$ The dominant process is the ejection of a single $1 \mathrm{~s}$ electron reflected by a main peak in the photoelectron spectrum. Smaller peaks or satellites appearing at higher ionization energy are associated with shake-up (or shake-off) processes, where, in addition to the $1 s$ hole, one or more valence electrons are excited. ${ }^{2,3}$ Typically, $30-40 \%$ of the $1 s$-photoionization cross section may be diverted to these shake-up (shake-off) states. Prominent satellites states at low energy with intensities up to $10 \%$ of that of the main peak arise from the $\pi-\pi^{*}$ excitations in simple unsaturated molecules like $\mathrm{N}_{2}$ and $\mathrm{CO}^{3}$

The availability of tunable synchrotron radiation has made it possible to study the dynamics of the photoionization process, that is, to map the spectral intensities (partial photoionization cross sections) of individual ionic states as a function of photon energy. ${ }^{4}$ In the $K$-shell region several such studies have been carried out for the $1 s$-hole main state of atoms ${ }^{5}$ and molecules. ${ }^{6,7}$ In the case of $\mathrm{N}_{2}$ and $\mathrm{CO}$, previously studied by electron energy loss ${ }^{8}$ and photoabsorption spectroscopy, ${ }^{9-11}$ the most conspicuous feature in the molecular photoelectron spectrum is the occurrence of $\sigma$-type shape resonances. In the case of $K$-shell satellites, where the lower intensities make experiments more difficult, synchrotron-radiation studies have been performed for the noble-gas atoms $\mathrm{He}$ and $\mathrm{Ar}$ (Ref. $5)$ and the $\mathrm{CO}$ molecule. ${ }^{12}$

A common feature of several approaches ${ }^{13-15}$ used in theoretical studies of molecular photoionization is that the photoelectron continuum is determined by a singleparticle (Hartree-Fock) equation with a suitably chosen potential for the electron-ion interaction. To date most applications have adopted the frozen-core Hartree-Fock (FCHF) model. In this approximation the neutralmolecule ground-state Hartree-Fock (HF) orbitals are assumed for both the initial and the final ionic states, and corresponding potentials defining the motion of the photoelectron in the field of the ion can readily be derived. The FCHF model neglects both the effects of relaxation, that is, the adjustment of the other electrons to the presence of the hole, and correlation.

Relaxation is particularly important in the case of $K$ shell ionization, often exceeding by far correlation corrections. Almost all previous studies of $K$-shell photoionization of molecules have been carried out in the FCHF approximation. ${ }^{16-19}$ While these studies proved quite successful in establishing and clarifying the occurrence of $\sigma$-type shape resonances in the $K$-shell single-hole photoionization cross section, the agreement with experimental resonance positions and widths was often less than satisfactory. Recently, Lynch and $\mathrm{McKoy}^{20}$ used a relaxedcore Hartree-Fock (RCHF) model to study the resonant $K$-shell photoionization cross section in $\mathrm{N}_{2}$. In this model the ionic state and the associated potential are expressed in terms of the "relaxed" orbitals generated by a separate HF calculation for the ionic state. This RCHF potential accounts for screening of the $1 s$ hole by the relaxation of the valence orbitals and is hence less attractive than the unscreened FCHF potential, leading to shifts of the resonance positions to higher energy. 
Indeed, for the $\mathrm{N}_{2} K$ shell ${ }^{20}$ the RCHF resonance position was 7-8 eV higher than the FCHF result and 3-4 $\mathrm{eV}$ above the experimental position. Furthermore, the broadening of the resonance in the RCHF description results in better agreement with the experimental cross sections than seen in the FCHF model.

Although the RCHF approximation maintains the simplicity of a single-particle model, the absence of orbital orthogonality introduces several complications into its application. First, in contrast to the FCHF case, the variational principle used to derive the ion-electron scattering equation does not impose orthogonality between the continuum and the relaxed open-shell orbital, resulting in a significantly more complicated equation for the continuum electron. Fortunately, this problem is not acute in the case of $K$-shell (main) ionization since, to a good approximation, the $1 s$-continuum nonorthogonality can be neglected. As we shall see, this is a consequence of the core-valence separation (CVS) associated with the large energy difference of $K$-shell and valence levels and of the weak coupling matrix elements for states with different $K$-shell occupation numbers. Secondly, the initial and final $N$-electron states are formulated in terms of mutually nonorthogonal sets of "relaxed" and "frozen" orbitals, complicating the evaluation of the $N$-electron transition moment. As a result the initial ground and final excited $N$-electron states lack strict orthogonality which introduces a dependence of the transition-moment matrix element on the origin of the molecular coordinate frame. This is clearly an artifact of the RCHF model. For $K$-shell ionization we will show that the CVS approximation can be used to eliminate in a well-defined manner these unphysical contributions. The remaining nonorthogonality contributions to the RCHF transition moment are, of course, meaningful and establish the improved level of description compared with the FCHF approximation.

Nonorthogonality of the relaxed continuum orbital and the frozen ground-state orbitals gives rise to specific contributions in the RCHF transition moment that contain continuum-bound overlap integrals. ${ }^{21}$ This contribution to the transition moment, referred to as the "conjugate" part, ${ }^{21,22}$ may be distinguished from the "direct" part containing continuum-bound dipole integrals. Obviously the relative importance of these two contributions depends on the photoelectron energy. In the high-energy or "sudden" limit ${ }^{23}$ the overlap integrals vanish much more rapidly than the dipole integrals and the transition moment is determined by the direct part. On the other hand, for low energies, the overlap contributions could become appreciable and substantially affect the photoionization cross section. The effect of these conjugate contributions may be more significant for satellite intensities than for single-hole cross sections, since selection rules for these differ from those of the direct part. ${ }^{21,22}$ As a consequence, satellites not detected at high photon energy might appear in a spectrum taken at near-threshold energy. Possible candidates for "conjugate shake-up" are, for example, the $2 p-3 s^{2} P$ satellites in the Ne $1 s$ photoelectron spectrum. ${ }^{5}$

To our knowledge, the role of the conjugate contribu- tion in molecular photoionization cross sections has never been investigated very quantitatively. Even for atoms, where more sophisticated methods are available ${ }^{24}$ the nature of these conjugate contributions is not fully understood. The striking discrepancy between theory and experiment seen in the $\mathrm{Ne} 1 s$ satellites referred to above serves as an example. ${ }^{5,25}$

The present study continues the previous work $^{20}$ on molecular $K$-shell ionization in the framework of the RCHF model. We present an alternative approach for evaluating the central quantity, i.e., the $N$-electron transition amplitude between the frozen ground and relaxed final states. The resulting expressions for both the single-hole and satellite states of the ion are more transparent and practical than the biorthogonal orbital formulations used previously. ${ }^{20}$ They allow us, in particular, to analyze and calculate separately the direct and conjugate contributions to the transition amplitudes and photoionization cross sections. The case of $K$-shell ionization of $\mathrm{CO}$ has been chosen for the present numerical studies. This choice focuses directly on $K$-shell relaxation and avoids possible complications arising from hole localization. In the present study we confine ourselves to the single-hole (main) states. Results for the $1 s$ satellites will be reported in a forthcoming publication. ${ }^{26}$

An outline of this paper is as follows. Section II discusses our formulation of the photoionization amplitudes in the RCHF model and the CVS approximation. The choice of single-particle scattering potentials to determine the photoelectron continua is reviewed in Sec. III. In Sec. IV we analyze the energies (resonance positions) and cross sections in the RCHF description in the framework of (low-order) perturbation theory. The computational details are given in Sec. V, while Sec. VI contains the discussion of our results and comparison to experiment. A summary of our findings and conclusions are given in Sec. VII.

\section{PHOTOIONIZATION CROSS SECTIONS IN THE FROZEN AND RELAXED HARTREE-FOCK APPROXIMATIONS}

\section{A. Final-state wave function}

The partial photoionization cross section at a photon energy $\omega$ leading to an ionic state $\left|\Psi_{n}^{N-1}\right\rangle$ and a photoelectron with kinetic energy $k^{2} / 2$ is given by the familiar golden-rule expression (in atomic units):

$$
\sigma_{n}(\omega)=\frac{4 \pi^{2}}{3 c} \omega \sum_{\mu, \lambda, v}\left|\left\langle\Psi_{n v, k \lambda}^{N}\left|\widehat{D}^{(\mu)}\right| \Psi_{0}^{N}\right\rangle\right|^{2} .
$$

Here $\left|\Psi_{n v, k \lambda}^{N}\right\rangle$ represents the final $N$-electron state describing asymptotically the ion in the state $\left|\Psi_{n v}^{N-1}\right\rangle$ and a photoelectron in the state $|k \lambda\rangle$. The additional quantum numbers $v$ and $\lambda$ specify degeneracies not observed in the experiment, e.g., magnetic spin quantum numbers, and angular momentum of the photoelectron. A nondegenerate initial (ground state) $\left|\Psi_{0}^{N}\right\rangle$ is assumed in Eq. (1), and $\widehat{D}^{(\mu)}, \mu=x, y, z$ denotes the components of the $N$ electron dipole operator 


$$
\widehat{\mathbf{D}}=\sum_{i=1}^{N} \mathbf{r}^{(i)}
$$

Energy conservation requires $k^{2} / 2=\omega-E_{n}^{N-1}+E_{0}^{N}$, where $E_{0}^{N}$ and $E_{n}^{N-1}$ are the energies of the initial ground state and the final ionic state, respectively. Rotational and vibrational degrees of freedom are suppressed in Eq. (1) and a fixed nuclear geometry is assumed for all electronic states.

To disentangle the many-body and continuum aspects in evaluating the bound-free $N$-electron transition amplitude

$$
A_{n v, k \lambda}^{(\mu)}=\left\langle\Psi_{n v, k \lambda}^{N}\left|\hat{D}^{(\mu)}\right| \Psi_{0}^{N}\right\rangle,
$$

a product approximation is normally assumed for the final state and $\left|\Psi_{n v, k \lambda}^{N}\right\rangle$ is written as the antisymmetrized product

$$
\left|\Psi_{n v, k \lambda}^{N}\right\rangle=c_{k \lambda}^{\dagger}\left|\Psi_{n v}^{N-1}\right\rangle
$$

of the ionic state $\left|\Psi_{n v}^{N-1}\right\rangle$ and the one-electron scattering orbital $\left|\psi_{k \lambda}^{(-)}\right\rangle$(with incoming-wave boundary conditions). In Eq. (4) we use the second-quantized notation, where $c_{k \lambda}^{\dagger}$ represents the creation operator associated with the continuum orbital $\left|\psi_{k \lambda}^{(-)}\right\rangle$. The form of Eq. (1) for the cross section assumes the final states to be normalized by

$$
\left\langle\Psi_{n v}^{N-1}\left|c_{k \lambda} c_{k^{\prime} \lambda}^{\dagger}\right| \Psi_{n v}^{N-1}\right\rangle=\delta\left(\frac{k^{\prime 2}}{2}-\frac{k^{2}}{2}\right) .
$$

To proceed we may insert the second-quantized representation of the dipole operator in Eq. (3) and make use of the commutation relations for (fermion) operators. For this purpose we use a representation in terms of neutral ground-state (frozen) Hartree-Fock orbitals $\left|\phi_{i}\right\rangle$ and the associated operators $a_{i}^{\dagger}\left(a_{i}\right)$ :

$$
\hat{D}^{(\mu)}=\sum_{i}\left\langle\phi_{i}\left|\hat{d}^{(\mu)}\right| \phi_{j}\right\rangle a_{i}^{\dagger} a_{j} .
$$

Since $c_{k}$ may be expressed as

$$
c_{k}=\sum_{i}\left\langle\psi_{k}^{(-)} \mid \phi_{i}\right\rangle a_{i}
$$

in terms of the operators $a_{i}$, the expression for the transition amplitude can be written $21,27,28$

$$
\begin{aligned}
A_{n, k}= & \left\langle\Psi_{n}^{N-1}\left|c_{k} \hat{D}\right| \Psi_{0}^{N}\right\rangle \\
= & \sum_{r}\left\langle\psi_{k}^{(-)}|\widehat{d}| \phi_{r}\right\rangle\left\langle\Psi_{n}^{N-1}\left|a_{r}\right| \Psi_{0}^{N}\right\rangle \\
& +\sum_{r}\left\langle\psi_{k}^{(-)} \mid \phi_{r}\right\rangle\left\langle\Psi_{n}^{N-1}\left|\hat{D} a_{r}\right| \Psi_{0}^{N}\right\rangle .
\end{aligned}
$$

Here and in the following the additional quantum numbers $\nu$ and $\lambda$ and the specification $\mu$ of the dipole operator are suppressed whenever they are not essential. According to Eq. (8), $A_{n, k}$ is the sum of two distinct parts $A_{n, k}^{\mathrm{I}}$ and $A_{n, k}^{\mathrm{II}}$. The first part $A_{n, k}^{\mathrm{I}}$, referred to as the direct part, combines the one-electron dipole matrix elements $\left\langle\psi_{k}^{(-)}|\hat{d}| \phi_{r}\right\rangle$ with the so-called spectroscopic amplitudes

$$
x_{r}^{(n)}=\left\langle\Psi_{n}^{N-1}\left|a_{r}\right| \Psi_{0}^{N}\right\rangle .
$$

The second term, on the other hand, contains products of the bound-continuum overlap integrals $\left\langle\psi_{k}^{(-)} \mid \phi_{r}\right\rangle$ and the bound-bound transition integrals

$$
y_{r}^{(n)}=\left\langle\Psi_{n}^{N-1}\left|\hat{D} a_{r}\right| \Psi_{0}^{N}\right\rangle .
$$

This contribution is the conjugate or nonorthogonality part; ${ }^{21,22}$ it only gives a contribution if the continuum orbital $\left|\psi_{k}^{(-)}\right\rangle$is not "orthogonal" to $\left|\Psi_{0}^{N}\right\rangle$, i.e., $\left\langle\Psi_{0}^{N}\left|c_{k}^{\dagger} c_{k}\right| \Psi_{0}^{N}\right\rangle \neq 0$. It is interesting to note that the direct and conjugate amplitudes impose different selection rules among the molecular orbitals, i.e., dipole and monopole rules, respectively. The energy dependence of the conjugate amplitude has not been directly calculated previously and should provide new insight into $K$-shell photoionization, especially in the context of satellite intensities. If the HF ground state

$$
\left|\Phi_{0}^{N}\right\rangle=\left|\phi_{1} \cdots \phi_{N}\right|
$$

is taken as the initial state, then the summation on the right-hand side of Eq. (8) runs over occupied orbitals $\left(n_{r}=1\right)$ only. If allowance for ground-state correlation is made, unoccupied orbitals $\left(\bar{n}_{r}=1-n_{r}=1\right)$ may also give contributions.

The simplest and most widely used approximation for the transition amplitudes of $\mathrm{Eq} .(8)$ is the frozen-core Hartree-Fock model. Here, besides using the HF approximation $\left|\Phi_{0}^{N}\right\rangle$ for the ground state, the ionic states are also expressed in terms of the "frozen" HF orbitals of the neutral ground state. Specifically, the states

$$
\left|\Phi_{h}^{N-1}\right\rangle=a_{h}\left|\Phi_{0}^{N}\right\rangle, \quad n_{h}=1
$$

and

$$
\left|\Phi_{j h l}^{N-1}\right\rangle=a_{j}^{\dagger} a_{h} a_{l}\left|\Phi_{0}^{N}\right\rangle, \quad \bar{n}_{j} n_{h} n_{l}=1
$$

are used to represent a single-hole (main) and a twohole-one-particle $(2 h-1 p)$ satellite state, respectively. Consistently defined "frozen" potentials (see Sec. III) are used to determine the continuum orbitals $\left|\psi_{k}^{(-)}\right\rangle$. These FCHF potentials impose orthogonality constraints ${ }^{10}$

$$
\left\langle\psi_{k}^{(-)} \mid \phi_{r}\right\rangle=0, \quad n_{r}=1
$$

between the continuum and the occupied HF orbitals, and consequently the conjugate part of the transition amplitude vanishes. For a single-hole (main) state the spectroscopic amplitudes of Eq. (9) are trivial,

$$
x_{r}^{(h)}=\left\langle\Phi_{0}^{N}\left|a_{h}^{\dagger} a_{r}\right| \Phi_{0}^{N}\right\rangle=\delta_{h r},
$$

so that the transition amplitude reduces to

$$
A_{h k}=\left\langle\psi_{k}^{(-)}|\hat{d}| \phi_{h}\right\rangle \text {. }
$$

For satellite states [Eq. (12b)] the FCHF model cannot be used, since both parts of the amplitude $A_{j h l, k}$ vanish. For the direct part this is so because the spectroscopic amplitudes vanish

$$
x_{r}^{(j h l)}=\left\langle\Phi_{0}^{N}\left|a_{l}^{\dagger} a_{h}^{\dagger} a_{j} a_{r}\right| \Phi_{0}^{N}\right\rangle=0 .
$$

For the conjugate part, on the other hand, we find nonvanishing moments, 


$$
\begin{aligned}
y_{r}^{(j h l)}=\left\langle\Phi_{0}^{N}\left|a_{l}^{\dagger} a_{h}^{\dagger} a_{j} \hat{D} a_{r}\right| \Phi_{0}^{N}\right\rangle \\
=\delta_{r l}\left\langle\phi_{j}|\hat{d}| \phi_{h}\right\rangle-\delta_{r h}\left\langle\phi_{j}|\hat{d}| \phi_{l}\right\rangle .
\end{aligned}
$$

However, the bound-free overlap integrals $\left\langle\psi_{k}^{(-)} \mid \phi_{r}\right\rangle$ vanish.

In the framework of many-body perturbation theory the FCHF model may be viewed as the zeroth-order approximation to the transition amplitudes (see Sec. IV B). Obviously one has to go beyond this level of description to account for relaxation and correlation. If one is only interested in the bound-bound amplitudes $x_{r}$ and $y_{r}$ one may, of course, resort to the well-developed methods of quantum chemistry. For the spectroscopic amplitudes $x_{r}$ direct computational schemes based on the one-particle Green's function have been widely applied. ${ }^{29}$ In the present study, rather than trying to obtain more accurate bound-bound amplitudes we are interested in a consistent treatment of the many-body and continuum aspects of the problem. Such a level of description, which goes beyond the FCHF model but maintains the essential simplifications of the single-particle picture, is provided by the relaxed-core Hartree-Fock approximation, discussed in Sec. II B.

\section{B. Spectroscopic amplitudes and conjugate transition moments in the RCHF approximation}

In the RCHF approximation distinct HF wave functions are used for the initial ground state and the final ionic state; a consistent relaxed ion core potential is used to determine the photoelectron continuum (see Sec. III). The lack of orthogonality between the frozen-core and relaxed-core HF orbitals leads to obvious complications. In the following we present a transparent and tractable approach allowing us, in particular, to distinguish readily between direct and conjugate contributions to the photoionization cross sections.

In addition to the FCHF orbitals $\left|\phi_{i}\right\rangle$ and the corresponding creation (destruction) operators $a_{i}^{\dagger}\left(a_{i}\right)$ considered in Sec. II A, we now introduce the set of relaxed orbitals $\left|\psi_{i}\right\rangle$ generated by an appropriate HF calculation for the ion. Denoting the associated creation (destruction) operators by $c_{i}^{\dagger}\left(c_{i}\right)$, the RCHF representation of a single-hole (main) state is

$$
\left|\Psi_{h}^{N-1}\right\rangle=c_{h}\left|\bar{\Phi}_{0}^{N}\right\rangle, \quad n_{h}=1
$$

where

$$
\left|\bar{\Phi}_{0}^{N}\right\rangle=\left|\psi_{1} \cdots \psi_{N}\right|
$$

is defined in analogy to $\left|\Phi_{0}^{N}\right\rangle$ as the Slater determinant of the lowest $N$ relaxed orbitals $\left|\psi_{i}\right\rangle$. To obtain the spectroscopic amplitude $x_{r}^{(h)}$ for the single-hole state of Eq. (17) and the uncorrelated ground state $\left|\Phi_{0}^{N}\right\rangle$ we have to evaluate the overlap integral between the two $(N-1)$ electron states $c_{h}\left|\bar{\Phi}_{0}^{N}\right\rangle$ and $a_{r}\left|\Phi_{0}^{N}\right\rangle$ represented by Slater determinants of mutually nonorthogonal sets of orbitals. As shown in the Appendix the result simply is

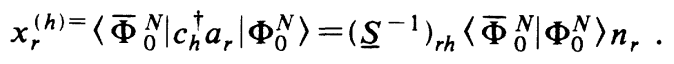

Here, $\underline{S}$ denotes the $N \times N$ matrix of the "relaxed-frozen" overlap integrals

$$
\begin{aligned}
& S_{i j}=\left\langle\psi_{i} \mid \phi_{j}\right\rangle, \quad n_{i}=n_{j}=1 \\
& \left\langle\bar{\Phi}_{0}^{N} \mid \Phi_{0}^{N}\right\rangle=\operatorname{det} \underline{S} .
\end{aligned}
$$

Similarly, one finds for the conjugate transition moment

$$
\begin{aligned}
y_{r}^{(h)}= & \left\langle\bar{\Phi}{ }_{0}^{N}\left|c_{h}^{\dagger} \hat{D}_{a_{r}}\right| \Phi_{0}^{N}\right\rangle \\
= & {\left[-\left(\underline{S}^{-1} \underline{d} \underline{S}^{-1}\right)_{r h}\right.} \\
& \left.+\left(\underline{S}^{-1}\right)_{r h} \operatorname{Tr}\left(\underline{d}^{-1}\right)\right]\left\langle\bar{\Phi}_{0}^{N} \mid \Phi_{0}^{N}\right\rangle_{n_{r},}
\end{aligned}
$$

where $\underline{d}$ denotes the $N \times N$ matrix of dipole integrals

$$
d_{i j}=\left\langle\psi_{i}|\hat{d}| \phi_{j}\right\rangle, \quad n_{i}=n_{j}=1
$$

for the occupied relaxed and frozen orbitals, and $\operatorname{Tr}(\underline{a})$ is the trace of $\underline{a}$. We note in passing that transition moments of this type are also encountered in the HF description of $x$-ray emission intensities. The final result for the single-hole state photoionization amplitude in the $\mathrm{RCHF}$ approximation is

$$
\begin{aligned}
& A_{h, k}=\sum_{r}\left\{\left\langle\psi_{k}^{(-)}|\hat{d}| \phi_{r}\right\rangle\left(\underline{S}^{-1}\right)_{r h}\right. \\
&+\left\langle\psi_{k}^{(-)} \mid \phi_{r}\right\rangle[-\left(\underline{S}^{-1} \underline{d} \underline{S}^{-1}\right)_{r h} \\
&\left.\left.+\left(\underline{S}^{-1}\right)_{r h} \operatorname{Tr}\left(\underline{d} \underline{S}^{-1}\right)\right]\right\}\left\langle\bar{\Phi}^{N} \mid \Phi_{0}^{N}\right\rangle .
\end{aligned}
$$

To identify clearly the major contributions in Eq. (24) we consider the simplified amplitude obtained by setting $\underline{S}=\underline{1}$ and $d_{i j}=\left\langle\phi_{i}|\hat{d}| \phi_{j}\right\rangle$ :

$$
\begin{aligned}
A_{h, k} \approx & \left\langle\psi_{k}^{(-)}|\hat{d}| \phi_{h}\right\rangle-\sum_{r}\left\langle\psi_{k}^{(-)} \mid \phi_{r}\right\rangle\left\langle\phi_{r}|\hat{d}| \phi_{h}\right\rangle n_{r} \\
& +\left\langle\psi_{k}^{(-)} \mid \phi_{h}\right\rangle \sum_{i}\left\langle\phi_{i}|\hat{d}| \phi_{i}\right\rangle n_{i} .
\end{aligned}
$$

The "diagonal" term in Eq. (25) depends on the origin of the molecular coordinates. As discussed in Sec. II C, this is due to spurious contributions which arise from the lack of strict orthogonality between the initial ground and the final excited state.

The $2 h-1 p$ satellite states are given in the RCHF approximation by

$$
\left|\Psi_{j h l}^{N-1}\right\rangle=c_{j}^{\dagger} c_{h} c_{l}\left|\bar{\Phi}_{0}^{N}\right\rangle, \quad \bar{n}_{j} n_{h} n_{l}=1 .
$$

The corresponding spectroscopic amplitudes $x_{r}^{(j h l)}$ and conjugate transition moments $y_{r}^{(j h l)}$ can be obtained analogously to the case of single-hole states (see Appendix). The resulting expressions are

$$
\begin{aligned}
x_{r}^{(j h l)}= & \left\langle\bar{\Phi}_{0}^{N}\left|c_{l}^{\dagger} c_{h}^{\dagger} c_{j} a_{r}\right| \Phi_{0}^{N}\right\rangle \\
= & {\left[\left(\underline{S}^{-1}\right)_{r l} \sum_{u}\left\langle\psi_{j} \mid \phi_{u}\right\rangle \underline{S}_{u h}^{-1}\right] } \\
& -[h \leftrightarrow l]]\left\langle\bar{\Phi}_{0}^{N} \mid \Phi_{0}^{N}\right\rangle n_{r}
\end{aligned}
$$

and 


$$
\begin{aligned}
& y_{r}^{(j h l)}=\left\langle\bar{\Phi}_{0}^{N}\left|c_{l}^{\dagger} c_{h}^{\dagger} c_{j} \hat{D} a_{r}\right| \Phi_{0}^{N}\right\rangle \\
& =\left[\left[\left(\underline{S}^{-1}\right)_{r l} \sum_{u}\left\langle\psi_{j}|\widehat{d}| \phi_{u}\right\rangle\left(\underline{S}^{-1}\right)_{u h}+\left(\underline{S}^{-1} \underline{d} \underline{S}^{-1}\right)_{r h} \sum_{u}\left\langle\psi_{j} \mid \phi_{u}\right\rangle\left(\underline{S}^{-1}\right)_{u l}\right.\right. \\
& \left.+\left(\underline{S}^{-1}\right)_{r l} \sum_{u, v}\left\langle\psi_{j} \mid \phi_{u}\right\rangle\left(\underline{S}^{-1}\right)_{u v}\left[\operatorname{Tr}\left(\widehat{d} \underline{S}^{-1}\right) \underline{1}-\underline{d} \underline{S}^{-1}\right]_{v h}\right]-[h \leftrightarrow l] \mid\left\langle\bar{\Phi}_{0}^{N} \mid \Phi_{0}^{N}\right\rangle n_{r},
\end{aligned}
$$

where $[h \leftrightarrow l]$ means repeating the preceding terms, but with $h$ and $l$ indices interchanged. Here, besides the occupied orbital overlap and dipole matrices $\underline{S}$ and $\underline{d}$, the overlap $\left\langle\psi_{j} \mid \phi_{r}\right\rangle$ and dipole $\left\langle\psi_{j}|\hat{d}| \phi_{r}\right\rangle$ integrals are required for the relaxed (unoccupied) orbital $\left|\psi_{j}\right\rangle$ of the satellite state and the frozen occupied orbitals $\left|\phi_{r}\right\rangle, r \leq N$. Again, the dominant direct and conjugate contributions to the satellite amplitude $A_{j h l, k}$ become apparent in the (zeroth-order) approximation $\underline{S}=\underline{1}$ and $d_{i j}=\left\langle\phi_{i}|\hat{d}| \phi_{j}\right\rangle$ :

$$
\begin{aligned}
A_{j h l, k}= & \left\langle\left\langle\psi_{k}^{(-)}|\hat{d}| \phi_{l}\right\rangle\left\langle\psi_{j} \mid \phi_{h}\right\rangle+\left\langle\psi_{k}^{(-)} \mid \phi_{l}\right\rangle\left\langle\psi_{j}|\hat{d}| \phi_{h}\right\rangle+\sum_{r}\left\langle\psi_{k}^{(-)} \mid \phi_{r}\right\rangle\left\langle\phi_{r}|\hat{d}| \phi_{h}\right\rangle\left\langle\psi_{j} \mid \phi_{l}\right\rangle n_{r}\right. \\
& \left.-\left\langle\psi_{k}^{(-)} \mid \phi_{l}\right\rangle \sum_{r}\left\langle\psi_{j} \mid \phi_{r}\right\rangle\left\langle\phi_{r}|\hat{d}| \phi_{h}\right\rangle n_{r}+\left\langle\psi_{k}^{(-)} \mid \phi_{l}\right\rangle\left\langle\psi_{j} \mid \phi_{h}\right\rangle \sum_{i}\left\langle\phi_{i}|\hat{d}| \phi_{l}\right\rangle n_{i}\right]-[h \leftrightarrow l] .
\end{aligned}
$$

It should be noted that the more intuitively derived expression for the conjugate shake-up amplitude ${ }^{22}$

$A^{\mathrm{II}}=\left\langle\psi_{k}^{(-)} \mid \phi_{l}\right\rangle\left\langle\psi_{j}|\hat{d}| \phi_{h}\right\rangle-\left\langle\psi_{k}^{(-)} \mid \phi_{h}\right\rangle\left\langle\psi_{j}|\hat{d}| \phi_{l}\right\rangle$

represents only a part of the conjugate contribution in Eq. (29).

\section{Nonorthogonality of initial and final states}

Complications arise in the RCHF approximation due to the lack of strict orthogonality between the $N$-electron ground state $\left|\Phi_{0}^{N}\right\rangle$ and the final (excited) states $c_{k}^{\dagger} c_{h}\left|\bar{\Phi}_{0}^{N}\right\rangle$ or $c_{k}^{\dagger} c_{j}^{\dagger} c_{h} c_{l}\left|\Phi_{0}^{N}\right\rangle$, respectively. In the case of a singlehole ionic core the overlap integral may be evaluated as follows:

$$
\left\langle\bar{\Phi}_{0}^{N}\left|c_{h}^{\dagger} c_{k}\right| \Phi_{0}^{N}\right\rangle=\sum_{r}\left\langle\psi_{k}^{(-)} \mid \phi_{r}\right\rangle x_{r}^{(h)}
$$

where the spectroscopic amplitudes $x_{r}^{(h)}$ are specified by Eq. (19). Similarly we find

$$
\left\langle\bar{\Phi}_{0}^{N}\left|c_{k} c_{j} c_{h}^{\dagger} c_{l}^{\dagger}\right| \Phi_{0}^{N}\right\rangle=\sum_{r}\left\langle\psi_{k}^{(-)} \mid \phi_{r}\right\rangle x_{r}^{(j h l)},
$$

in the case of a $2 h-1 p$ satellite; the spectroscopic amplitudes $x_{r}^{(j h l)}$ are given by Eq. (27). As a consequence of this nonorthogonality the dipole transition amplitudes $A_{n, k}$ [Eq. (3)] depend on the choice of origin of the molecular coordinate frame. In our explicit RCHF expressions [Eqs. (24), (25), and (27)-(29)] this becomes apparent through the occurrence of origin-dependent integrals such as $\left\langle\psi_{k}^{(-)}|\hat{d}| \phi_{r}\right\rangle$ or $\left\langle\psi_{r}|\hat{d}| \phi_{r}\right\rangle$. Clearly such contributions bear no physical meaning and are a mere artifact of the RCHF model. How can one distinguish between these artificial contributions to the transition amplitude and the genuine conjugate contributions in the RCHF approximation? In general, the answer is by no means clear. In the case of $K$-shell ionization, however, on which we shall focus here, the artificial nonorthogonality contributions can be discarded in a well-defined way as discussed in Sec. II D.

\section{Core-valence separation approximation}

Specialization to the case of $K$-shell ionization allows for a considerable simplification of the RCHF transition amplitudes. This is achieved by adopting the so-called core-valence separation approximation in which one neglects the coupling of states with different $1 s$ occupations. Formally this decoupling is introduced by neglecting the following types of Coulomb integrals: ${ }^{30}$

$$
\begin{aligned}
& V_{c v v^{\prime} v^{\prime \prime}}=V_{v c c^{\prime} v^{\prime \prime}}=\cdots=0, \\
& V_{c c^{\prime} c^{\prime \prime} v}=V_{c c^{\prime} v c^{\prime \prime}}=\cdots=0, \\
& V_{c c^{\prime} v v^{\prime}}=V_{v v^{\prime} c c^{\prime}}=0,
\end{aligned}
$$

where $c, c^{\prime}$, and $c^{\prime \prime}$ and $v, v^{\prime}$, and $v^{\prime \prime}$ label $K$-shell and valence orbitals, respectively. The errors introduced here in the wave functions and energies are of the order of $V / \Delta \varepsilon$ and $V^{2} / \Delta \varepsilon$, respectively, where $V$ stands for one of the neglected Coulomb integrals (32) and $\Delta \varepsilon$ is the corevalence energy separation. To see the effect of the corevalence separation on the RCHF amplitudes, consider the relaxed core orbital $\left|\psi_{c}\right\rangle$. The perturbation expansion of $\left|\psi_{c}\right\rangle$ in terms of the frozen orbitals $\left|\phi_{r}\right\rangle$ through first order,

$$
\left|\psi_{c}\right\rangle=\left|\phi_{c}\right\rangle+\sum_{\substack{r \\ r \neq c}} \frac{\gamma V_{c c c r}}{\varepsilon_{c}-\varepsilon_{r}}\left|\phi_{r}\right\rangle+O(2),
$$

shows that the relaxed orbital differs from the frozen one by the admixture of valence orbitals, the mixing coefficients being of the order of $V_{c c c r} /\left(\varepsilon_{r}-\varepsilon_{c}\right)$. In Eq. (33) $\gamma$ is a constant, depending on the specific RCHF treatment. Obviously, in the CVS approximation, the relaxed- and frozen-core orbitals are identical (up to an eventual phase factor) leading to the following simplifications for the relaxed-frozen overlap integrals:

$$
\begin{aligned}
& \left|\left\langle\psi_{c} \mid \phi_{c}\right\rangle\right|=1, \\
& \left\langle\psi_{r} \mid \phi_{c}\right\rangle=0, \quad r \neq c \\
& \left\langle\psi_{k}^{(-)} \mid \phi_{c}\right\rangle=0 .
\end{aligned}
$$

An immediate consequence of the CVS approximation is that the initial-final state overlaps in Eqs. (31a) and (31b), vanish, and there are no longer artificial contributions in the transition amplitudes. Applying the CVS approximation Eq. (34) to the amplitude $A_{c, k}$ for a $K$-shell single- 
hole state $c^{-1}$, the RCHF result of Eq. (24) becomes

$$
\begin{aligned}
A_{c, k}=\left\langle\left\langle\psi_{k}^{(-)}|\hat{d}| \phi_{c}\right\rangle-\sum_{r, r^{\prime}}\left\langle\psi_{k}^{(-)} \mid \phi_{r}\right\rangle\left(\underline{S}^{-1}\right)_{r, r^{\prime}}\right. \\
\\
\left.\times\left\langle\psi_{r^{\prime}}|\hat{d}| \phi_{c}\right\rangle\right]\left\langle\bar{\Psi}_{0}^{N} \mid \Phi_{0}^{N}\right\rangle .
\end{aligned}
$$

as can be readily shown, the quantity

$$
\hat{P}=\sum_{r, r^{\prime}}\left|\phi_{r}\right\rangle\left(\underline{S}^{-1}\right)_{r, r^{\prime}}\left\langle\psi_{r^{\prime}}\right|
$$

is a non-Hermitian projection operator, and using this definition the amplitude $A_{c, k}$ may be written as

$$
A_{c, k}=\left\langle\psi_{k}^{(-)}|(1-\hat{P}) \hat{d}| \phi_{c}\right\rangle\left\langle\Phi_{0}^{N} \mid \Phi_{0}^{N}\right\rangle .
$$

Comparing this result with the direct amplitude,

$$
A_{c, k}^{\mathrm{I}}=\left\langle\psi_{k}^{(-)}|\hat{d}| \phi_{c}\right\rangle\left\langle\bar{\Phi}_{0}^{N} \mid \Phi_{0}^{N}\right\rangle,
$$

we see that the effect of the conjugate contribution is to project out a certain part of the continuum orbital, namely, its occupied frozen-orbital components, as can be seen from the zeroth-order approximation to the projector [Eq. (36)],

$$
\hat{\boldsymbol{P}}^{(0)}=\sum_{r}\left|\phi_{r}\right\rangle\left\langle\phi_{r}\right| n_{r} .
$$

A further understanding of the contributions to the $\mathrm{RCHF}$ amplitude will be gained from the perturbation point of view given in Sec. IV B.

In the case of a $2 h-1 p$ satellite $\left(j^{1}, c^{-1}, l^{-1}\right)$ where $c$ denotes a core orbital, the general RCHF result [Eqs. (8), (27), and (28)] simplifies considerably in the CVS approximation [Eq. (34)] and the final result for the amplitude $A_{j c l, k}$ can be put into the compact form

$$
\begin{aligned}
\boldsymbol{A}_{j c l, k}=[ & -\left\langle\psi_{k}^{(-)}|(1-\hat{\boldsymbol{P}}) \hat{d}| \phi_{c}\right\rangle\left\langle\psi_{j} \mid \phi_{l}\right\rangle^{\mathrm{eff}} \\
& \left.+\left\langle\psi_{j}|(1-\hat{\boldsymbol{P}}) \hat{d}| \phi_{c}\right\rangle\left\langle\psi_{k}^{(-)} \mid \phi_{l}\right\rangle^{\mathrm{eff}}\right]\left\langle\bar{\Phi}_{0}^{N} \mid \Phi_{0}^{N}\right\rangle .
\end{aligned}
$$

Here $\widehat{P}$ is given by Eq. (36) and the "effective" overlaps are defined:

$$
\begin{aligned}
& \left\langle\psi_{j} \mid \phi_{l}\right\rangle^{\mathrm{eff}}=\sum_{r}\left\langle\psi_{j} \mid \phi_{r}\right\rangle\left(\underline{S}^{-1}\right)_{r l}, \\
& \left\langle\psi_{k}^{(-)} \mid \phi_{l}\right\rangle^{\mathrm{eff}}=\sum_{r}\left\langle\psi_{k}^{(-)} \mid \phi_{r}\right\rangle\left(\underline{S}^{-1}\right)_{r l} .
\end{aligned}
$$

The direct amplitude above is given by

$$
\left.A_{j c l, k}^{\mathrm{I}}=\left\langle\psi_{k}^{(-)}|\hat{d}| \phi_{c}\right\rangle\left(-\left\langle\psi_{j} \mid \phi_{l}\right\rangle\right\rangle^{\mathrm{eff}}\left\langle\bar{\Phi}_{0}^{N} \mid \Phi_{0}^{N}\right\rangle\right),
$$

where the factor

$$
x_{c}^{(j c l)}=-\left\langle\psi_{j} \mid \phi_{l}\right\rangle^{\mathrm{eff}}\left\langle\bar{\Phi}_{0}^{N} \mid \Phi_{0}^{N}\right\rangle
$$

is readily identified as the spectroscopic satellite amplitude. Comparison of the direct and the full amplitudes clarifies the effect of the conjugate part: except for introducing the projection operator $\hat{P}$ in the direct part, there arises an additional term formally obtained by exchanging the role of $j$ and $k$ in the first ("effective" direct) part $\left(A_{j c l, k}^{\mathrm{I}} \rightarrow A_{k c l, j}^{\mathrm{I}}\right)$. We may call this latter part the "effective" conjugate shake-up amplitude. It should be noted that the quantity

$$
A_{c, j}=\left\langle\psi_{j}|(1-\hat{P}) \hat{d}| \phi_{c}\right\rangle\left\langle\bar{\Phi}_{0}^{N} \mid \Phi_{0}^{N}\right\rangle
$$

represents the transition moment for a bound $K$-shell single excitation $\left(j^{1} c^{-1}\right)$ in the CVS approximation.

The expressions (35) - (40) for $K$-shell ionization amplitudes in the RCHF and CVS approximations are still in terms of (primitive) spin-orbital representations. The construction of spin-free equations for ionic states of proper spatial and spin symmetry is straightforward. The resulting expression for the $1 s$-hole main state in the $1 s$ photoionization of $\mathrm{CO}$ will be given in Sec. V. The case of $K$-shell satellites will be discussed in a forthcoming publication. ${ }^{26}$

\section{POTENTIALS FOR $\boldsymbol{K}$-SHELL PHOTOELECTRONS}

Single-particle scattering equations may be derived from the variational expression ${ }^{31}$

$$
\left\langle\delta \Psi^{N}|\hat{H}-E| \Psi^{N}\right\rangle=0,
$$

where $\left|\Psi^{N}\right\rangle$ is an $N$-electron final state expressed in terms of target-state orbitals that are kept fixed and a continuum orbital $\left|\psi_{k}^{(-)}\right\rangle$which is to be determined. Here the variation $\left|\delta \Psi^{N}\right\rangle$ arises from the variation of the continuum orbital $\left|\delta \psi_{\mathbf{k}}\right\rangle$ only. In the FCHF model for the photoionization of a closed-shell molecule the final state (with $S=0$ ) corresponding to a single-hole ionic state

$$
\left|\Phi_{h \gamma}^{N-1}\right\rangle=a_{h \gamma}\left|\Phi_{0}^{N}\right\rangle
$$

may be written as

$$
\left|\Psi_{h, \mathbf{k}}^{N}\right\rangle=\frac{1}{\sqrt{2}}\left(a_{\mathbf{k} \alpha}^{\dagger} a_{h \alpha}+a_{\mathbf{k} \beta}^{\dagger} a_{h \beta}\right)\left|\Phi_{0}^{N}\right\rangle,
$$

where the second-quantized notation introduced in Sec. II $\mathrm{A}$ is used and $\alpha$ and $\beta$ are the usual spin states. The analogous RCHF state reads

$$
\left|\Psi_{h, \mathbf{k}}^{N}\right\rangle=\frac{1}{\sqrt{2}}\left(c_{\mathbf{k} \alpha}^{\dagger} c_{h \alpha}+c_{\mathbf{k} \beta}^{\dagger} c_{h \beta}\right)\left|\bar{\Phi}_{0}^{N}\right\rangle .
$$

In either case the result of the variation of Eq. (45) takes on the form ${ }^{15}$

$$
\begin{aligned}
& \left(\hat{t}+\sum_{\substack{i \\
i \neq h}}\left(2 \widehat{J}_{i}-\widehat{K}_{i}\right) n_{i}+\widehat{J}_{h}+\widehat{K}_{h}+E_{0}^{N}-\varepsilon_{h}-E\right)|\mathbf{k}\rangle+|h\rangle\langle h \mid \mathbf{k}\rangle\left(E_{0}^{N}-2 \varepsilon_{h}+J_{h h}-E\right) \\
& +|h\rangle\left\langle h\left|\hat{t}+\sum_{\substack{i \\
i \neq h}}\left(2 \widehat{J}_{i}-\widehat{K}_{i}\right) n_{i}\right| \mathbf{k}\right\rangle+\left(\hat{t}+\sum_{\substack{i \\
i \neq h}}\left(2 \widehat{J}_{i}-\widehat{K}_{i}\right) n_{i}\right)|h\rangle\langle h \mid \mathbf{k}\rangle=0,
\end{aligned}
$$


where the notation $|i\rangle$ applies to the frozen orbitals $\left|\phi_{i}\right\rangle$ or the relaxed orbitals $\left|\psi_{i}\right\rangle$, respectively. In Eq. (47) $\hat{t}$ represents the kinetic energy and the electron-nuclei attraction, $\widehat{J}_{i}$ and $\widehat{K}_{i}$ denote Coulomb and exchange operators associated with the orbitals $|i\rangle$. The energy $\varepsilon_{h}$ of the open-shell orbitals is given by

$$
\varepsilon_{h}=\left\langle h\left|\hat{t}+\sum_{i}\left(2 \widehat{J}_{i}-\widehat{R}_{i}\right) n_{i}\right| h\right\rangle,
$$

which still applies if $|h\rangle$ is not an eigenfunction of the inserted HF operator. Furthermore,

$$
J_{h h}=\left\langle h\left|\widehat{J}_{h}\right| h\right\rangle-\left\langle h\left|\widehat{K}_{h}\right| h\right\rangle
$$

is the Coulomb self-energy of the orbital $|h\rangle$ and

$$
E_{0}^{N}=\langle N|\hat{H}| N\rangle
$$

represents the (first-order) energy of the frozen $\left(|N\rangle=\left|\Phi_{0}^{N}\right\rangle\right)$ or relaxed $\left(|N\rangle=\left|\Phi_{0}^{N}\right\rangle\right) \quad N$-electron ground state. In deriving Eq. (47) orthogonality was assumed between the continuum orbital $|\mathbf{k}\rangle$ and the doubly occupied orbitals $|r\rangle, n_{r}=1, r \neq h$, but no such restriction with respect to the open-shell orbital $|h\rangle$ was imposed on $|\mathbf{k}\rangle$.

To discuss the complications introduced by the possible lack of orthogonality between the orbitals $|\mathbf{k}\rangle$ and $|h\rangle$ we project both sides of Eq. (47) on $|h\rangle$, yielding the equation

$$
\begin{aligned}
\left\langle h\left|\hat{t}+\sum_{\substack{i \\
i \neq h}}\left(2 \widehat{J}_{i}-\widehat{K}_{i}\right) n_{i}+\widehat{J}_{h}\right| \mathbf{k}\right\rangle & \\
& +\left(E_{0}^{N}-\varepsilon_{h}-E\right)\langle h \mid \mathbf{k}\rangle=0 .
\end{aligned}
$$

In the FCHF model, since $|h\rangle$ is an eigenfunction of the (ground-state) HF operator, this equation reduces to ${ }^{15}$

$$
0=\left(E_{0}^{N}-E\right)\langle h \mid \mathbf{k}\rangle=\left(\varepsilon_{h}-\frac{1}{2} k^{2}\right)\langle h \mid \mathbf{k}\rangle,
$$

where the second equation follows by writing the total energy $E$ as

$$
E=E_{0}^{N}-\varepsilon_{h}+\frac{1}{2} k^{2}
$$

Obviously, Eq. (52) requires $\langle h \mid \mathbf{k}\rangle=0$ for positive energies $\frac{1}{2} k^{2}$ and with this orthogonality constraint one obtains the familiar improved virtual orbital (IVO) equation $^{32}$

$$
\left[\hat{t}+\sum_{\substack{i \\ i \neq h}}\left(2 \widehat{J}_{i}-\widehat{K}_{i}\right) n_{i}+\widehat{J}_{h}+\widehat{K}_{h}-\frac{1}{2} k^{2}\right)|\mathbf{k}\rangle=0 .
$$

It should be noted that the orthogonality properties,

$$
\langle\mathbf{k} \mid i\rangle=0, \quad n_{i}=1
$$

are not automatically fulfilled by the solution of Eq. (54) but rather must be introduced as an additional constraint.

Turning to the case of the RCHF model we write the continuum function as

$$
|\mathbf{k}\rangle=\left|\mathbf{k}^{\prime}\right\rangle+\langle h \mid \mathbf{k}\rangle|h\rangle,
$$

where $\left\langle h \mid \mathbf{k}^{\prime}\right\rangle=0$. Equation (51) can then be transformed to the following equation:

$$
\langle h \mid \mathbf{k}\rangle=\frac{\left\langle h\left|\hat{t}+\sum_{\substack{i \\ i \neq h}}\left(2 \widehat{J}_{i}-\widehat{K}_{i}\right) n_{i}+\widehat{J}_{h}\right| \mathbf{k}^{\prime}\right\rangle}{\varepsilon_{h}-\frac{1}{2} k^{2}}
$$

for the bound-free overlap $\langle h \mid \mathbf{k}\rangle$. In general, the numerator on the right-hand side of Eq. (56) does not vanish and one is faced with the situation that the variational principle explicitly requires a nonvanishing overlap $\langle h \mid \mathbf{k}\rangle$. Besides the much more complicated coupled equations that may result from Eq. (47) for $\left|\mathbf{k}^{\prime}\right\rangle$ and $\langle h \mid \mathbf{k}\rangle$, it is by no means clear how a properly normalized $N$-particle final state could be constructed in this case.

Fortunately, specialization to $K$-shell ionization allows one to get rid of these complications to a very good approximation. If $|\boldsymbol{h}\rangle=|c\rangle$ is a $K$-shell orbital the absolute value of the denominator in Eq. (56) is of the order of the core-valence energy gap. The matrix elements appearing in the numerator are small since they are of the mixed core-valence type $t_{c v}$ and $V_{c v v^{\prime} v^{\prime \prime}}$. Hence only a very small error is introduced by setting $\langle h \mid \mathbf{k}\rangle=0$, i.e., by adopting the strict core-valence separation approximation discussed in Sec. II D. Thus in the RCHF model of $K$-shell photoionization we may safely adopt the IVO Eq. (54).

\section{ANALYSIS OF THE RCHF MODEL}

\section{A. Resonance positions through second order of perturbation theory}

In the FCHF description of shape-resonant $K$-shell photoionization the potential does not include the effect of screening of the $1 s$ hole by the reorganization (relaxation) of the valence electrons. The FCHF potential is hence too attractive and the resulting resonance positions are usually several $\mathrm{eV}$ below the experimental values. A substantial shifting to higher energies has indeed been found by Lynch and $\mathrm{McKoy}{ }^{20}$ for the $\sigma^{*}$ shape resonance in $\mathrm{N}_{2}$ by allowing for electronic relaxation through the RCHF potential. While the shape of the resonance is now in much better agreement with the experiment the calculated resonance position lies a few eV above the experimental value. This discrepancy in resonance position was tentatively attributed to an "overscreening" introduced by the RCHF potential. To clarify this concept and to get a better understanding of the frozen- and relaxed-core approximations we compare the resonance energies in the FCHF and RCHF models with the exact result through second order of perturbation theory.

We specifically consider the energy $E_{v c}^{N}$ of a singlet core excitation $\left|\Psi_{v c}^{N}\right\rangle$ obtained from the unperturbed singly excited state

$$
\left|\Phi_{v c}^{N}\right\rangle=\frac{1}{\sqrt{2}}\left(a_{v \alpha}^{\dagger} a_{c \alpha}+a_{v \beta}^{\dagger} a_{c \beta}\right)\left|\Phi_{0}^{N}\right\rangle
$$

Here $|v\rangle$ represents an unoccupied (valence) orbital, e.g., $\pi^{*}$ or $\sigma^{*}$, and $c$ a $1 s$ orbital. The term value

$$
R_{v}=E_{v c}^{N}-E_{c}^{N-1}
$$

i.e., the energy with respect to that of the $1 s$-hole state,

$$
\left|\Psi_{c \gamma}^{N-1}\right\rangle \leftarrow a_{c \gamma}\left|\Phi_{0}^{N}\right\rangle,
$$


can be positive or negative. In the former case the term value is related to a resonance position, while in the latter it refers to the position of a bound state below the $1 s$ ionization threshold. All perturbation expansions used in the following are defined with respect to the ground-state HF representation.

Let us begin with the FCHF model. Here the term value $R_{v}^{\mathrm{FC}}$ is given by the eigenvalue $\varepsilon_{v}^{\mathrm{FC}}$ of the FCHF Eq. (54):

$$
\hat{f}^{\mathrm{FC}}\left|v^{\mathrm{FC}}\right\rangle=\varepsilon_{v}^{\mathrm{FC}}\left|v^{\mathrm{FC}}\right\rangle \text {. }
$$

The FCHF operator $\hat{f}^{\mathrm{FC}}$ can be written as

$$
\hat{f}^{\mathrm{FC}}=\hat{f}_{0}-\hat{J}_{c}+2 \hat{K}_{c},
$$

where

$$
\hat{f}_{0}=\hat{t}+\sum_{i}\left(2 \widehat{J}_{i}-\hat{K}_{i}\right) n_{i}
$$

is the ground-state HF operator. The form of the righthand side of Eq. (61) makes the perturbation expansions for $\left|v^{\mathrm{FC}}\right\rangle$ and $\varepsilon_{v}^{\mathrm{FC}}$ obvious:

$$
\begin{aligned}
\left|v^{\mathrm{FC}}\right\rangle=|v\rangle & +\sum_{\substack{q \\
q \neq v}} \frac{-V_{v c q c}+2 V_{v c c q}}{\varepsilon_{v}-\varepsilon_{q}} \bar{n}_{q}|q\rangle+O(2) \\
R_{v}^{\mathrm{FC}}=\varepsilon_{v}^{\mathrm{FC}}= & \varepsilon_{v}-J_{v c}+2 K_{v c}+2 K_{v c} \\
& +\sum_{\substack{q \neq v \\
\varepsilon_{v}-\varepsilon_{q}}} \frac{\left(V_{v c q c}-2 V_{v c c q}\right)^{2} \bar{n}_{q}}{\varepsilon} \\
& +O(2)+O(3)
\end{aligned}
$$

The direct part $\left(-J_{v c}\right)$ of the first-order contribution to the orbital energy accounts for the lowering of the electrostatic repulsion upon removal of a $1 s$ electron. The second-order term for the energy (and the first-order term for the wave function) reflects the adjustment (relaxation) of the relevant orbital $|v\rangle$ to the $1 s$ vacancy. It should be noted that the perturbation expansions (62) already include the effect of the orthogonality constraints $\left\langle v^{\mathrm{FC}} \mid r\right\rangle=0, n_{r}=1$ of the FCHF model by restricting the summations to unoccupied orbitals $|q\rangle$.

In the RCHF model too, the term value $R_{v}^{\mathrm{RC}}$ is given by the single particle energy $\varepsilon_{v}^{\mathrm{RC}}$. The RCHF operator

$$
\hat{f}^{\mathrm{RC}}=\hat{t}+\sum_{\substack{i \\ i \neq h}}\left(2 \widehat{J}_{i}^{\prime}-\hat{K}_{i}^{\prime}\right) n_{i}+\widehat{J}_{c}^{\prime}+\hat{K}_{c}^{\prime}
$$

has the same form as the FCHF operator but - as indicated by the primes - the Coulomb and exchange operators here are defined with respect to the relaxed orbitals $\left|\psi_{i}\right\rangle$ of a HF calculation for the ion. To derive the desired perturbation expansions we decompose the RCHF operator according to

$$
\begin{aligned}
\hat{f}^{\mathrm{RC}}= & \hat{f}_{0}+\hat{f}_{I}^{\mathrm{RC}}, \\
\hat{f}_{I}^{\mathrm{RC}}= & -\hat{J}_{c}+2 \hat{k}_{c}+\sum_{\substack{i \\
i \neq c}}\left(2 \hat{J}_{i}^{\prime}-\hat{K}_{i}^{\prime}-2 \hat{J}_{i}+\hat{K}_{i}\right) n_{i} \\
& +\hat{J}_{c}^{\prime}+K_{c}^{\prime}-\hat{J}_{c}-\hat{K}_{c},
\end{aligned}
$$

where one has to take into account that the perturbation part $\hat{f}_{I}^{\mathrm{RC}}$ itself has its perturbation expansion

$$
\hat{f}_{I}^{\mathrm{RC}}=\hat{f}_{I}^{\mathrm{RC}}(0)+\hat{f}_{I}^{\mathrm{RC}}(1)+\cdots
$$

The zeroth-order term is clearly identified as

$$
\hat{f}_{I}^{\mathrm{RC}}(0)=-\hat{J}_{c}+2 \hat{K}_{c}
$$

and the first-order term may be easily evaluated once the ionic HF operator generating the relaxed orbitals has been specified. Obviously this operator may deviate from the IVO form of Eq. (54); it may even be the case that the relaxed orbitals are calculated by a generalized HF procedure where the operator approach does not apply at all. In any case, we may assume the following form for the expansion of the relaxed orbitals through first order

$\left|\psi_{j}\right\rangle=|j\rangle+\sum_{\substack{q \\ q \neq j}} \frac{-V_{j c q c}+\gamma V_{j c c q}}{\varepsilon_{j}-\varepsilon_{q}}|q\rangle+O(2)$

where $\gamma$ is a parameter, depending on the choice of the ionic HF equations. Now the perturbation expansion of the excited RCHF orbital $\left|v^{\mathrm{RC}}\right\rangle$ may be written as

$$
\begin{aligned}
\left|v^{\mathrm{RC}}\right\rangle=|v\rangle & +\sum_{\substack{q \\
q \neq v}} \frac{-V_{v c q c}+2 V_{v c c q}}{\varepsilon_{v}-\varepsilon_{q}} \bar{n}_{q}|q\rangle \\
& +\sum_{\substack{q \\
q \neq v}} \frac{-V_{v c q c}+\gamma V_{v c c q}}{\varepsilon_{v}-\varepsilon_{q}} n_{q}|q\rangle+O(2) .
\end{aligned}
$$

Here, in addition to the first-order FCHF form, admixtures of occupied orbitals $|q\rangle$ occur. Note that in the latter part the exchange terms have the factor $\gamma$ instead of 2; this guarantees the orthogonality constraints $\left\langle v^{\mathrm{RC}} \mid \psi_{j}\right\rangle=0$ for the occupied relaxed orbitals $\left|\psi_{j}\right\rangle$ [Eq. (67)] through first order. For the RCHF energy $\varepsilon_{v}^{\mathrm{RC}}$ the second-order expansion

$$
\begin{aligned}
R_{v}^{\mathrm{RC}}=\varepsilon_{v}^{\mathrm{RC}}= & \varepsilon_{v}^{\mathrm{FC}}(2)+\sum_{q} \frac{\left(-V_{v c q c}+\gamma V_{v c c q}\right)^{2}}{\varepsilon_{v}-\varepsilon_{q}} n_{q} \\
& +\left\langle v\left|\hat{f}_{I}^{\mathrm{RC}}(1)\right| v\right\rangle+O(3)
\end{aligned}
$$

comprises the FCHF result $\varepsilon_{v}^{\mathrm{FC}}(2)$ [Eq. (62b)] and two additional terms, the first of which is associated with the occupied-orbital admixtures to $|v\rangle$. The other term reads

$$
\begin{aligned}
&\left\langle v\left|\hat{f}_{I}^{\mathrm{RC}}\right| v\right\rangle= \sum_{\substack{r \\
r \neq c}}\left\langle v\left|2 \hat{J}_{r}^{\prime}-\hat{K}_{r}^{\prime}-2 \hat{J}_{r}+\hat{K}_{r}\right| v\right\rangle n_{r} \\
&=2 \sum_{\substack{r, q \\
r \neq c}} \frac{\left(-V_{r c q c}+\gamma V_{r c c q}\right)\left(2 V_{v r v q}-V_{v r q v}\right)}{\varepsilon_{r}-\varepsilon_{q}} \\
& \times n_{r} \bar{n}_{q},
\end{aligned}
$$

where real-valued Coulomb integrals have been assumed for simplicity. This term has the obvious physical meaning of a screening energy for the orbital $|v\rangle$, since it accounts for the change in the electrostatic respulsion between the orbital $|v\rangle$ and the occupied valence orbitals upon relaxation of the latter. It should be noted that in the core-valence separation approximation, which for simplicity will also be adopted in the present perturbation 
studies, $\left|\psi_{c}\right\rangle=|c\rangle$ and hence the part $\widehat{J}_{c}^{\prime}+\widehat{K}_{c}^{\prime}-\widehat{J}_{c}-\widehat{K}_{c}$ of $\hat{f}_{I}^{\mathrm{RC}}$ vanishes.

Now we may compare the FCHF and RCHF approximations with the exact term values $R_{v}$ through second order. The second-order expansion of the energy of the excited state $\left|\Psi_{v c}^{N}\right\rangle$ has the following form:

$$
\begin{aligned}
E_{v c}^{N}(2)= & E_{0}^{N}(1)+\varepsilon_{v}-\varepsilon_{c}-J_{v c}+2 K_{v c}+U_{v c}^{(2)}(p-h) \\
& +U_{v c}^{(2)}(2 p-2 h)+U_{v c}^{(2)}(3 p-3 h) .
\end{aligned}
$$

Here $E_{0}^{N}(1)=\left\langle\Phi_{0}^{N}|\hat{H}| \Phi_{0}^{N}\right\rangle$ is the ground-state HF energy. The three terms $U_{v c}^{(2)}(p-h), U_{v c}^{(2)}(2 p-2 h)$, and $U_{v c}^{(2)}(3 p-$ $3 h$ ) represent the second-order contributions arising from the interaction of $\left|\Phi_{c v}^{N}\right\rangle$ with (other) $p$ - $h$ states, $2 p-2 h$ states, and $3 p-3 h$ states, respectively. Similarly, we find for the energy of the ionic state the expansion

$$
\begin{aligned}
E_{c}^{N-1}(2)= & E_{0}^{N}(1)-\varepsilon_{c}+U_{c}^{(2)}(2 h-1 p) \\
& +U_{c}^{(2)}(3 h-2 p),
\end{aligned}
$$

where $U_{c}^{(2)}(2 h-1 p)$ and $U_{c}^{(2)}(3 h-2 p)$ arise from the interaction of the single-hole state with $2 h-1 p$ and $3 h-2 p$ states, respectively. Combining the expansions (70) and (71) yields for the term value

$$
\begin{aligned}
R_{v}(2)= & \varepsilon_{v}-J_{v c}+2 K_{v c}+U_{v c}^{(2)}(p-h)+U_{v c}^{(2)}(2 p-2 h) \\
& -U_{c}^{(2)}(2 h-1 p)+U_{v c}^{(2)}(3 p-3 h)-U_{c}^{(2)}(3 h-2 p) .
\end{aligned}
$$

The second-order contributions appearing here have been specified elsewhere ${ }^{33,34}$ in the case of primitive (spinorbital) excitations. Here we have to consider the somewhat more complicated spin-free expressions appropriate for a singlet excitation (57) and a doublet ionic state. Again using the core-valence approximation we find

$$
U_{v c}^{(2)}(p-h)=\sum_{\substack{q \\ q \neq v}} \frac{\left(V_{v c q c}-2 V_{v c c q}\right)^{2}}{\varepsilon_{v}-\varepsilon_{q}} \bar{n}_{q} .
$$

This result is readily identified as the second-order contribution of the FCHF energy (62b). The contribution

$U_{c}^{(2)}(2 h-1 p)$

$$
=-\sum_{r, q} \frac{2 V_{c r c q}^{2}+2 V_{c r q c}^{2}-2 V_{c r c q} V_{c r q c}}{\varepsilon_{q}-\varepsilon_{r}} \bar{n}_{q} n_{r}
$$

represents the second-order relaxation energy of the ionic state. For discussion of the $2 p-2 h$ contribution $U_{c v}^{(2)}(2 p-$ $2 h)$ it is useful to split it into a part $U_{v c}^{\prime}(2 p-2 h)$ arising from excitations of the form $(q)^{1}(v)^{1}(c)^{-1}(r)^{-1}$ and a remainder $U_{c v}^{\prime \prime}(2 p-2 h)$. The former part, being the essential one, can be brought into the following form:

$$
\begin{aligned}
& U_{c v}^{\prime}(2 p-2 h)=Q+S+P, \\
& Q=-2 \sum_{\substack{q, r \\
q \neq v}} \frac{q}{\varepsilon_{q}-\varepsilon_{r}}\left(V_{c r c q}^{2}-V_{c r c q} V_{c r q c}+V_{c r q c}^{2}\right) n_{r} \bar{n}_{q} \\
&-\sum_{r} \frac{1}{\varepsilon_{v}-\varepsilon_{r}}\left(V_{c r c v}+V_{c r v c}\right)^{2} n_{r},
\end{aligned}
$$

$$
\begin{aligned}
S= & 2 \sum_{\substack{q, r \\
q \neq v}} \frac{1}{\varepsilon_{q}-\varepsilon_{r}}\left(2 V_{c r c q} V_{v r v q}-V_{c r c q} V_{v r q v}\right. \\
& \left.-V_{c r q c} V_{v r v q}+2 V_{c r q c} V_{v r q v}\right) n_{r} \bar{n}_{q} \\
+2 & \sum_{r} \frac{1}{\varepsilon_{v}-\varepsilon_{r}}\left(V_{c r c v}+V_{c r v c}\right) V_{v r v v} n_{r}, \\
P= & -2 \sum_{q, r} \frac{1}{\varepsilon_{q}-\varepsilon_{r}}\left(V_{v r v q}-V_{v r v q} V_{v r q v}+V_{v r q v}^{2}\right) n_{r} \bar{n}_{q} \\
& -\sum_{r} \frac{1}{\varepsilon_{v}-\varepsilon_{r}} V_{v r v v}^{2} n_{r} .
\end{aligned}
$$

Each of these parts has a distinct physical meaning. The first part $Q$ is identical with the $1 s$ relaxation energy (74), except for the term in which $q=v$. With respect to the term value this means that the $1 s$ relaxation energy largely cancels out, the remainder being

$Q-U_{c}^{(2)}(2 h-1 p)=\sum_{r} \frac{V_{c r c v}^{2}-4 V_{c r c v} V_{c r v c}+V_{c r v c}^{2}}{\varepsilon_{v}-\varepsilon_{r}} n_{r}$.

Up to terms with exchange integrals, this part is retrieved in the RCHF energy $E_{v}^{\mathrm{RC}}$, namely, in the second term on the right-hand side of Eq. (69a). The next part $S$ in Eq. (75) obviously recovers the screening energy (69b) introduced by the RCHF approximation. It should, however, be noted that there remain discrepancies with respect to exchange contributions irrespective of the choice of $\gamma$ [Eq. (67)] adopted for the relaxed orbitals. Neither the FCHF nor the RCHF approximation includes the third part $P$ accounting for the polarization of the ionic core by the bound or free electron in the orbital $v$. The energy associated with the polarization is clearly negative, shifting the term value or the resonance position to lower energy.

The remaining second-order contributions are the remainder of the $2 p-2 h$ part ( $\mathscr{E}$ denotes the exchange energy)

$U_{c v}^{\prime \prime}(2 p-2 h)=-\sum_{\substack{r, q, q^{\prime} \\ q \leq q^{\prime}(\neq v)}} \frac{2 V_{r v q q^{\prime}}^{2}+\mathscr{E}}{\varepsilon_{q}+\varepsilon_{q^{\prime}}-\varepsilon_{r}-\varepsilon_{v}} n_{r} \bar{n}_{q} \bar{n}_{q}$,

and the difference

$$
\begin{aligned}
U_{c v}^{(2)}(3 h-2 p)-U_{c}^{(2)}(3 h-2 p) & \\
= & +\sum_{\substack{r, r^{\prime} \\
r \leq r^{\prime}}} \frac{2 V_{v q r r^{\prime}}^{2}+\mathscr{E}}{\varepsilon_{v}+\varepsilon_{q}-\varepsilon_{r}-\varepsilon_{r^{\prime}}} \bar{n}_{q} n_{r} n_{r^{\prime}}
\end{aligned}
$$

mainly reflecting the difference of correlation energies in the excited and the ionic states. Both contributions are absent in the single-particle approximations but are expected to be only of minor importance.

We can summarize our results as follows. Of all second-order contributions the FCHF energy includes only the Tamm-Dancoff (TDA) term $\left[U_{v c}^{(2)}(p-h)\right]$ arising from the mixing with other single excitations. The FCHF result (in second order) differs from the RCHF energy by the screening energy (69b) and the occupied orbital analogous to $U_{v c}^{(2)}(p-h)$, that is, 


$$
\sum_{q} \frac{\left(V_{v c q c}-\gamma V_{v c c q}\right)^{2} n_{q}}{\varepsilon_{v}-\varepsilon_{q}}
$$

Both contributions are positive, explaining the higher RCHF energies. In the exact second-order result they are retrieved (up to exchange terms) as the energy $S$ [Eq. (75b)] and the difference $Q-U_{c}^{(2)}(2 h-1 p)$ [Eq. (76)] of excited- and ionic-state relaxation energies. The major deficiency of the RCHF model appears to be the neglect of the polarization energy (75d) leading to too high term values or resonance positions.

\section{B. Transition amplitudes}

In the preceding section, Sec. IV A, we adopted perturbation theory to analyze resonance positions or term values in $K$-shell photoexcitation. We now look at the corresponding transition amplitudes in light of perturbation theory. Again, we consider the singlet $K$-shell hole state $\left|\Psi_{v c}^{N}\right\rangle$ resulting from the zeroth-order state $\left|\Phi_{v c}^{N}\right\rangle$ of Eq. (57), where $|v\rangle$ represents an unoccupied valence or continuum orbital. The expansion of the transition amplitude $A_{c, v}=\left\langle\Psi_{v c}^{N}|\hat{D}| \Psi_{0}^{N}\right\rangle$ [see Eq. (3)] through first order is

$$
\begin{aligned}
A_{c, v}(1)= & \sqrt{2}\langle v|\hat{d}| c\rangle+\left\langle\Psi_{v c}^{(1)}|\hat{D}| \Phi_{0}^{N}\right\rangle \\
& +\left\langle\Phi_{v c}^{N}|\hat{D}| \Psi_{0}^{(1)}\right\rangle
\end{aligned}
$$

where $\left|\Psi_{v c}^{(1)}\right\rangle$ and $\left|\Psi_{0}^{(1)}\right\rangle$ represent the first-order excited and ground states, respectively. The first-order contribution,

$$
\left\langle\Psi_{v c}^{(1)}|\hat{D}| \Phi_{0}^{N}\right\rangle=\sqrt{2} \sum_{\substack{q \\ q \neq v}} \frac{-V_{v c q c}+2 V_{v c c q}}{\varepsilon_{v}-\varepsilon_{q}} \bar{n}_{q}\langle q|\hat{d}| c\rangle,
$$

arises from the admixture of other single excitations $\left|\Phi_{q c}^{N}\right\rangle$ (adopting here again the CVS approximation). This part of the first-order amplitude is properly accounted for by the Tamm-Dancoff approximation to electronic excitation. $^{35}$ The other first-order contribution in Eq. (79), associated with ground-state correlation, or more precisely, with the admixture of double excitations to the HF ground state, is included in the random-phase approximation $^{35}$ (RPA) which provides a consistent firstorder description of the single-excitation transition amplitudes; for a more detailed discussion see, e.g., Ref. 33. In the present context it is important to realize that for $K$-shell excitations in the CVS approximation the RPA reduces to the TDA and the ground-state correlation contribution $\left\langle\Phi_{v c}^{N}|\hat{D}| \Psi_{0}^{(1)}\right\rangle$ in Eq. (79) vanishes.

We can now compare the full first-order result (in the CVS approximation) with the RCHF amplitude $A_{c, v}^{\mathrm{RC}}$ [Eqs. (35) or (40)]. To first order and in spin-free notation we find

$$
\begin{aligned}
A_{c, v}^{\mathrm{RC}}(1)=\sqrt{2} & \langle v|\hat{d}| c\rangle+\left\langle v^{(1)}|\hat{d}| c\right\rangle \\
& \left.-\sum_{r}\left\langle v^{(1)} \mid r\right\rangle\left\langle r|\hat{d}|_{c}\right\rangle_{n_{r}}\right] .
\end{aligned}
$$

Obviously, the conjugate part cancels all contributions arising from the occupied orbitals in the first-order term of $\left|v^{\mathrm{RC}}\right\rangle$ [Eq. (70)], i.e.,

$$
\begin{aligned}
\left|v^{(1)}\right\rangle-\sum_{r}|r\rangle\left\langle r \mid v^{(1)}\right\rangle & \\
& =\sum_{\substack{q \\
q \neq v}} \frac{-V_{v c q c}+2 V_{v c c q}}{\varepsilon_{v}-\varepsilon_{q}} \bar{n}_{q}|q\rangle,
\end{aligned}
$$

and the final result is seen to be identical with the full (first-order) amplitude. Thus we have arrived at the important conclusions that the RCHF description of single photoexcitation amplitudes in the $K$-shell regime is complete (up to terms vanishing in the CVS approximation) through first order of perturbation theory, and is thus comparable to the level of the RPA treatment. Interestingly, the FCHF amplitudes $A_{c, v}^{\mathrm{FC}}$ are also consistent through first order, since, due to the orthogonality constraints $\left\langle v^{\mathrm{FC}} \mid r\right\rangle=0, n_{r}=1$, the first-order term of $\left|v^{\mathrm{FC}}\right\rangle$ [Eq. (62a)] contains only unoccupied orbitals $|q\rangle$.

\section{v. Calculations}

The bound electronic wave functions required in the present applications were obtained from Hartree-Fock calculations for the ${ }^{1} \Sigma^{+}$ground state of $\mathrm{CO}$ $\left(1 \sigma^{2} 2 \sigma^{2} 3 \sigma^{2} 4 \sigma^{2} 1 \pi^{4} 5 \sigma^{2}\right)$ and for the C $1 s$ and $\mathrm{O} 1 s$ hole states of $\mathrm{CO}^{+}$. The SCF basis used in these calculations consisted of the $7 s / 4 p / 2 d$ contraction of $13 s / 8 p / 2 d$ Cartesian Gaussians. The exponents and contraction coefficients (No. 6.72.2 for $\mathrm{C}$ and No. 8.76.2 for $\mathrm{O}$ ) were taken from Huzinaga. ${ }^{36}$ The exponents for the $d$ functions were 0.92 and 0.256 for $C$, and 1.324 and 0.445 for $O$. The calculations were done at the equilibrium nuclear geometry of 2.132 a.u. For the ground state of the neutral molecule an SCF energy of -112.7844 a.u. was obtained; the energies for the $\mathrm{C} 1 s$ and $\mathrm{O} 1 s$ hole states were -101.8625 and -92.8818 a.u., respectively. The overlap integrals $S_{i j}$ [Eq. (20)] and dipole integrals $d_{i j}$ [Eq. (23)] between the frozen (ground-state HF) and the relaxed (ionic HF) orbitals were calculated using the single-center expansion techniques described below. The results are listed in Tables I and II.

The methods for obtaining the photoelectron continuum wave functions and bound-continuum integrals have been used in many previous applications and are amply described in Refs. 15 and 37 . In brief, the continuum function is written as the partial-wave expansion

$$
\psi_{\mathbf{k}}^{(-)}(\mathbf{r})=\left(\frac{2}{\pi}\right)^{1 / 2} \sum_{l, m} \frac{i^{l}}{k} \psi_{k l m}^{(-)}(\mathbf{r}) Y_{l m}^{*}(\hat{\mathbf{k}}) .
$$

Each partial wave $\psi_{k l m}(\mathbf{r})$ satisfies the LippmannSchwinger equation

$$
\psi_{k l m}^{(-)}(\mathbf{r})=\phi_{k l m}^{c}+G_{c}^{(-)}\left(\hat{v}+\frac{1}{r}\right) \psi_{k l m} .
$$

Here $G_{c}^{(-)}$is the Coulomb Green's function with incoming-wave boundary conditions and $\hat{v}$ is the staticexchange potential for the molecular ion [see Eq. (54)] taken either in the frozen core $\left(\hat{v}=\hat{v}^{\mathrm{FC}}\right)$ or the relaxed core $\left(\hat{v}=\hat{v}^{\mathbf{R C}}\right)$ representation. Orthogonalization be- 
TABLE I. Overlap integrals $S_{i j}$ [Eq. (20)]. These overlaps contain an arbitrary phase due to the sign of the wave function.

\begin{tabular}{|c|c|c|c|c|c|c|}
\hline \multicolumn{7}{|l|}{ Relaxed } \\
\hline & & & C $1 s(2 \sigma)^{\mathrm{a}}$ & & & \\
\hline $1 \sigma$ & 0.999 & $-0.905 \times 10^{-5}$ & $-0.177 \times 10^{-3}$ & $-0.381 \times 10^{-3}$ & $0.107 \times 10^{-3}$ & \\
\hline $2 \sigma$ & $-0.877 \times 10^{-5}$ & -0.998 & $0.622 \times 10^{-2}$ & $0.688 \times 10^{-2}$ & $-0.700 \times 10^{-2}$ & \\
\hline $3 \sigma$ & $-0.393 \times 10^{-3}$ & $-0.498 \times 10^{-2}$ & -0.994 & $0.887 \times 10^{-1}$ & $-0.142 \times 10^{-1}$ & \\
\hline $4 \sigma$ & $0.569 \times 10^{-3}$ & $-0.431 \times 10^{-2}$ & $0.906 \times 10^{-1}$ & 0.988 & $0.747 \times 10^{-1}$ & \\
\hline $5 \sigma$ & $-0.149 \times 10^{-3}$ & $-0.341 \times 10^{-2}$ & $-0.196 \times 10^{-1}$ & $-0.799 \times 10^{-1}$ & 0.976 & \\
\hline \multirow[t]{2}{*}{$1 \pi$} & & & & & & 0.986 \\
\hline & & & $\mathrm{O} 1 s(1 \sigma)^{\mathrm{b}}$ & & & \\
\hline $1 \sigma$ & 0.999 & $-0.696 \times 10^{-4}$ & $0.749 \times 10^{-2}$ & $0.372 \times 10^{-2}$ & $0.866 \times 10^{-3}$ & \\
\hline $2 \sigma$ & $-0.693 \times 10^{-4}$ & -0.999 & $0.150 \times 10^{-3}$ & $0.242 \times 10^{-3}$ & $0.107 \times 10^{-2}$ & \\
\hline $3 \sigma$ & $0.561 \times 10^{-2}$ & $0.229 \times 10^{-3}$ & -0.995 & $0.125 \times 10^{-1}$ & $-0.480 \times 10^{-1}$ & \\
\hline $4 \sigma$ & $-0.232 \times 10^{-2}$ & $0.225 \times 10^{-3}$ & $0.732 \times 10^{-2}$ & 0.981 & -0.142 & \\
\hline $5 \sigma$ & $-0.656 \times 10^{-3}$ & $0.137 \times 10^{-2}$ & $-0.521 \times 10^{-1}$ & 0.147 & 0.981 & \\
\hline $1 \pi$ & & & & & & -0.972 \\
\hline
\end{tabular}

${ }^{a}$ The frozen orbitals are from the neutral ground state. The relaxed orbitals are from the ion state, $2 \sigma^{-1}$, i.e., the C $1 s^{-1}$ state of $\mathrm{CO}^{+}$.

${ }^{\mathrm{b}}$ The same as footnote a except the relaxed orbitals are from the ion state $1 \sigma^{-1}$, i.e., the $\mathrm{O} 1 s^{-1}$ state of $\mathrm{CO}^{+}$.

tween the continuum and the occupied orbitals is taken into account by means of the Philips-Kleinman pseudopotential as described in Ref. 15. The solution of the Lippmann-Schwinger equations $(84)$ is based on the Schwinger variational method. By assuming a separable approximation to the potential,

$$
\widehat{V}=\hat{v}+\frac{1}{r},
$$

of the form

$$
\hat{V} \cong \hat{V}^{s}=\sum_{i, j}\left\langle\mathbf{r}|\hat{V}| \alpha_{i}\right\rangle\left(\underline{V}^{-1}\right)_{i j}\left\langle\alpha_{j}|\boldsymbol{V}| \mathbf{r}^{\prime}\right\rangle,
$$

the solutions of the integral equations (84) can be written as

$$
\begin{aligned}
\psi_{k l m}^{(-)}(\mathbf{r})=\phi_{k l m}^{c}(\mathbf{r})+\sum_{i, j} & \left\langle\mathbf{r}\left|G_{c}^{(-)} \hat{V}\right| \alpha_{i}\right\rangle\left(\underline{D}^{-1}\right)_{i j} \\
& \times\left\langle\alpha_{j}|\hat{V}| \phi_{k l m}^{c}\right\rangle .
\end{aligned}
$$

Here $\underline{V}$ and $\underline{D}$ denote matrices with matrix elements

$$
V_{i j}=\left\langle\alpha_{i}|\hat{V}| \alpha_{j}\right\rangle
$$

and

\begin{tabular}{|c|c|c|c|c|c|c|}
\hline Relaxed & $1 \sigma$ & $2 \sigma$ & $3 \sigma$ & $4 \sigma$ & $5 \sigma$ & $1 \pi$ \\
\hline & & & $\mathrm{C} 1 s(2 \sigma)$ & & & \\
\hline $1 \sigma$ & $0.913^{\mathrm{a}}$ & $0.437 \times 10^{-3}$ & $-0.181 \times 10^{-1}$ & $0.472 \times 10^{-1}$ & $-0.234 \times 10^{-1}$ & $-0.511 \times 10^{-1}$ \\
\hline $2 \sigma$ & $-0.114 \times 10^{-2}$ & 1.216 & $0.370 \times 10^{-1}$ & $-0.204 \times 10^{-1}$ & $0.853 \times 10^{-1}$ & $-0.494 \times 10^{-1}$ \\
\hline $3 \sigma$ & $0.202 \times 10^{-1}$ & $0.499 \times 10^{-1}$ & -0.257 & -0.595 & -0.107 & 0.604 \\
\hline $4 \sigma$ & $0.440 \times 10^{-1}$ & $0.176 \times 10^{-1}$ & 0.705 & 0.879 & -0.905 & -0.126 \\
\hline $5 \sigma$ & $-0.268 \times 10^{-1}$ & $0.697 \times 10^{-1}$ & $-0.103 \times 10^{-2}$ & -0.885 & -1.551 & 0.297 \\
\hline \multirow[t]{2}{*}{$1 \pi$} & $0.489 \times 10^{-1}$ & $-0.581 \times 10^{-1}$ & 0.625 & $0.160 \times 10^{-1}$ & -0.329 & 0.283 \\
\hline & & & $\mathrm{O} 1 s(1 \sigma)$ & & & \\
\hline $1 \sigma$ & 0.912 & $-0.100 \times 10^{-3}$ & $-0.144 \times 10^{-1}$ & $-0.595 \times 10^{-1}$ & $-0.328 \times 10^{-1}$ & $-0.605 \times 10^{-1}$ \\
\hline $2 \sigma$ & $-0.506 \times 10^{-3}$ & 1.216 & $0.369 \times 10^{-1}$ & $-0.188 \times 10^{-1}$ & $0.563 \times 10^{-1}$ & $-0.389 \times 10^{-1}$ \\
\hline $3 \sigma$ & $0.260 \times 10^{-1}$ & $0.358 \times 10^{-1}$ & -0.355 & -0.617 & $-0.243 \times 10^{-1}$ & 0.592 \\
\hline $4 \sigma$ & $0.571 \times 10^{-1}$ & $0.243 \times 10^{-1}$ & 0.621 & 1.002 & -0.588 & -0.108 \\
\hline $5 \sigma$ & $-0.205 \times 10^{-1}$ & $-0.549 \times 10^{-1}$ & 0.168 & -0.735 & -1.742 & 0.327 \\
\hline $1 \pi$ & $-0.668 \times 10^{-1}$ & $0.283 \times 10^{-1}$ & -0.600 & -0.143 & 0.183 & -0.532 \\
\hline
\end{tabular}

$$
D_{i j}=\left\langle\alpha_{i}\left|\hat{V}-\hat{V} G_{c}^{(-)} \hat{V}\right| \alpha_{j}\right\rangle \text {. }
$$

TABLE II. Dipole integrals $d_{i j}$ [Eq. (23)] between frozen and relaxed orbitals in a.u. For the $\sigma \rightarrow \sigma, \pi \rightarrow \pi$ transition $\widehat{d}=z$; for $\sigma \rightarrow \pi, \hat{d}=(x-i y) / \sqrt{2}$; and for $\pi \rightarrow \sigma, \hat{d}=-(x+i y) / \sqrt{2}$.

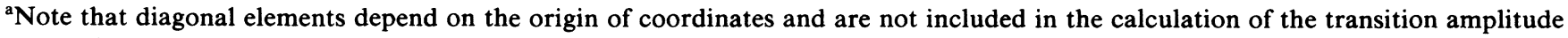
due to the CVS approximation discussed in Sec. II D. 
The set of functions $\left|\alpha_{i}\right\rangle$ referred to as the scattering basis set consists of suitably chosen discrete functions such as Cartesian Gaussian functions. The scattering basis set used in the present calculations is specified in Table III.

All matrix elements and functions arising in the computation of the partial wave $\psi_{\mathrm{klm}}^{(-)}$according to Eqs. (87)-(89) are evaluated using single-center expansions about the center of gravity. The details of these expansions are as follows.

(i) Maximum angular momentum in the expansion of the occupied orbitals in the direct potential is 29 .

(ii) Maximum angular momentum in the expansion of the occupied orbitals in the exchange potential is $25(1 \sigma)$,
$25(2 \sigma), 15(3 \sigma), 15(4 \sigma), 10(5 \sigma)$, and $10(1 \pi)$.

(iii) Maximum angular momentum in the expansion of $1 / r_{12}$ in the direct and exchange terms is 58 and 29 , respectively.

(iv) All other single-center expansions were truncated at $l=29$.

The partial-wave expansion of the continuum orbital, Eq. (83), was truncated at $l_{P}=8$. The resulting radial integrals were obtained using a Simpson-rule quadrature. The grid contained 500 points and extended to $69.0 \mathrm{a} . \mathrm{u}$. with a step size of 0.01 a.u. near the nuclei and a maximum step size of 0.40 a.u.

Following Eq. (35) we introduce the spin-free transition amplitudes $I_{l m \mu}$ for the partial waves $\psi_{k l m}$

$$
\begin{aligned}
I_{l m \mu} & =\frac{1}{\sqrt{2}}\left(A_{1 s \alpha, k l m \beta}^{(\mu)}+A_{1 s \beta, k l m \alpha}^{(\mu)}\right) \\
& =\sqrt{2}\left\langle\bar{\Phi}_{0}^{N} \mid \Phi_{0}^{N}\right\rangle\left\langle\left\langle\psi_{k l m}^{(-)}\left|\widehat{r}_{\mu}\right| \phi_{1 s}\right\rangle-\sum_{r, r^{\prime}}\left\langle\psi_{k l m}^{(-)} \mid \phi_{r}\right\rangle\left(\underline{S}^{-1}\right)_{r r^{\prime}}\left\langle\psi_{r^{\prime}}\left|\hat{r}_{\mu}\right| \phi_{1 s}\right\rangle\right)
\end{aligned}
$$

Here, the dipole operator components

$$
\widehat{r}_{\mu}=\left\{\begin{array}{l}
\mp(\hat{x} \pm i \hat{y}) / \sqrt{2} \text { for } \mu= \pm 1, \\
\hat{z} \text { for } \mu=0
\end{array}\right.
$$

are used and $\left|\phi_{1 s}\right\rangle$ refers to the core orbital C $1 s(2 \sigma)$ or $\mathrm{O} 1 s(1 \sigma)$. In Eq. (90b) only spin-free (spatial) quantities are retained, and, in particular, $\underline{S}$ represents the matrix of spatial relaxed-frozen overlap integrals $\left\langle\psi_{r} \mid \phi_{r^{\prime}}\right\rangle$. The $N$ electron overlap $\left\langle\bar{\Phi}_{0}^{N} \mid \Phi_{0}^{N}\right\rangle$ is given by the square of $\operatorname{det}(\underline{S})$. The decomposition of the full amplitude $I_{l m \mu}$ into the direct $I^{(\mathrm{I})}$ and conjugate contribution $I^{(\mathrm{II})}$,

$$
I_{l m \mu}=I_{l m \mu}^{(\mathrm{I})}+I_{l m \mu}^{(\mathrm{II})}
$$

according to Eq. (90b), is obvious. The dipole integrals $\left\langle\psi_{k l m}\left|\hat{r}_{\mu}\right| \phi_{1 s}\right\rangle$ and overlaps $\left\langle\psi_{k l m} \mid \phi_{r}\right\rangle$ were calculated with the single-center expansion technique specified above. The total photoionization cross section for the $1 s$-hole main state averaged over all molecular orientations is obtained from the partial amplitudes (90b) by

$$
\sigma=\frac{4 \pi^{2}}{3 c} \omega k \sum_{l, m, \mu}\left|I_{l m \mu}\right|^{2}
$$

The corresponding asymmetry parameter $\beta$ of the photoelectron angular distribution (with respect to the polar-

TABLE III. Scattering basis sets used in Eq. (87).

\begin{tabular}{ccc}
\hline $\begin{array}{c}\text { Continuum } \\
\text { symmetry }\end{array}$ & $\begin{array}{c}\text { Type of Gaussian } \\
\text { function }^{\mathrm{a}}\end{array}$ & Exponents \\
\hline \multirow{2}{*}{$\sigma$} & Cartesian $s$ & $10.0,4.0,1.5,0.5,0.1$ \\
& Cartesian $z$ & $2.0,1.0,0.5,0.1$ \\
$\pi$ & Cartesian $\rho_{x}$ & $10.0,4.0,1.5,0.5,0.1$ \\
& Cartesian $d_{x z}$ & $1.0,0.1$ \\
\hline \hline
\end{tabular}

${ }^{\text {a }}$ Cartesian functions are centered on each nucleus. For details of the forms of these functions and their use see Ref. 15. ization of the light) is given by

$$
\begin{aligned}
\beta=\frac{4 \pi^{2}}{5 c \sigma} k \sum_{\substack{l, m, \mu \\
l^{\prime}, m^{\prime}, \mu^{\prime} \\
\mu^{\prime \prime}}} & (-1)^{m-\mu^{\prime}} I_{l m \mu} I_{l^{\prime} m^{\prime} \mu^{\prime}}^{*}[(2 l+1) \\
& \left.\times\left(2 l^{\prime}+1\right)\right]^{1 / 2}\langle 1100 \mid 20\rangle\left\langle l l^{\prime} 0 \mid 20\right\rangle \\
& \times\left\langle 11-\mu \mu^{\prime} \mid 2 \mu^{\prime \prime}\right\rangle\left\langle l l^{\prime}-m-m^{\prime} \mid 2-\mu^{\prime \prime}\right\rangle .
\end{aligned}
$$

Here $\left\langle l_{1} m_{1} l_{2} m_{2} \mid l m\right\rangle$ is the Clebsch-Gordan coefficient $\left(l_{1} m_{1} l_{2} m_{2} \mid l_{1} l_{2} l m\right)$ in the notation of Edmonds. ${ }^{38}$

\section{RESULTS AND DISCUSSION}

\section{A. C (1s) ionization}

Figure 1 shows the shape-resonant $\mathrm{C} 1 s$ photoionization cross section in $\mathrm{CO}$ calculated both in the FCHF and the RCHF approximations, together with experimental results by Kay et al. ${ }^{39}$ and by Truesdale et al. ${ }^{6}$ As for $\mathrm{N}_{2}$, the two results are significantly different. Without valence-electron relaxation one finds a relatively narrow and intense peak about $5 \mathrm{eV}$ above the $\mathrm{C} 1 s$ ionization threshold (296.2 eV). The relaxed description (RCHF), on the other hand, results in a substantial lowering, broadening, and shifting of the $\sigma^{*}$ resonance centered at $11.2 \mathrm{eV}$ above threshold. This difference is clearly an effect of the screening of the $1 s$ hole by the relaxed valence electrons leading to a much less attractive potential than the "unscreened" potential of the FCHF model. The experimental resonance position, somewhat obscured by autoionization structures, appears to be between 8 and $9 \mathrm{eV}$, that is $2-3 \mathrm{eV}$ below our RCHF result. According to the discussion in Sec. IV A the overshooting of the calculated resonance position seems to be primarily induced by the neglect of target polarization in the present ap- 


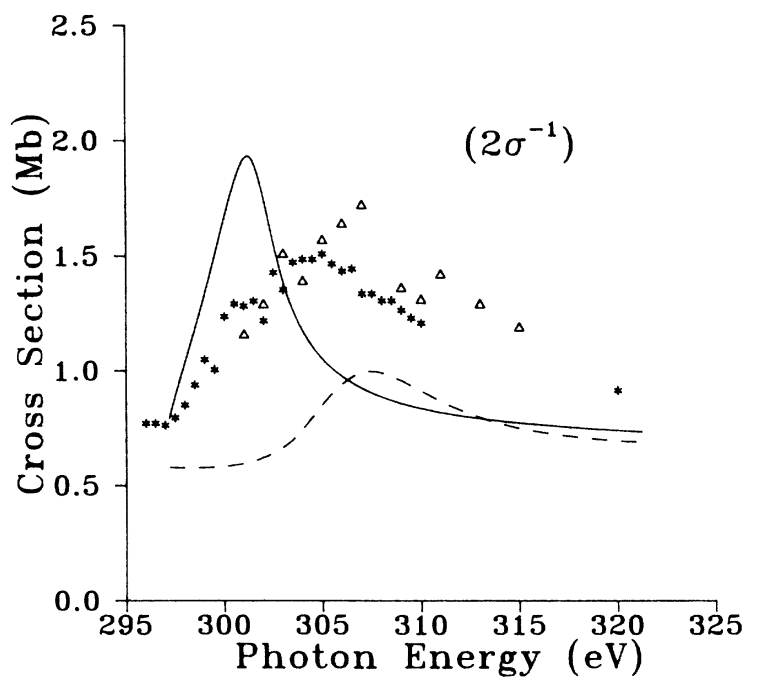

FIG. 1. Photoionization cross sections leading to the $2 \sigma^{-1}$ (C $1 s^{-1}$ ) state of $\mathrm{CO}^{+}$. — , present results (length) in the FCHF approximation; - - - present results (length) in the RCHF approximation; *, experimental results of Ref. 39; $\triangle$, experimental results of Ref. 6.

proach. Inclusion of the attractive polarization potential not only would lower the resonance position but would also enhance the resonance peak.

It may be worth noting that the different magnitudes in the frozen and relaxed cross sections result to some extent from the absence of the overall spectroscopic factor

$$
\left|x_{1 s}\right|^{2}=\left|\left\langle\bar{\Phi}_{0}^{N} \mid \Phi_{0}^{N}\right\rangle\right|^{2},
$$

in the expression for the FCHF cross section. This quantity specifies the amount of spectral strength diverted from the single-hole state to shake-up and shake-off states. The present RCHF calculations give a value of $\left|x_{1 s}\right|^{2}=0.79$ which is slightly larger than the result $(0.68)$ found in a recent Green's-function study. ${ }^{40}$

In Fig. 2 we compare our calculated frozen- and relaxed-core photoionization cross sections with the frozen-core results obtained previously by the StieltjesTschebycheff moment theory ${ }^{18}$ (STMT) and by the multiple scattering (MS) method. ${ }^{41}$ The present frozen-core curves should be essentially identical to those of the STMT studies, since the underlying single-particle scattering potential is the same. Nevertheless, we see drastic discrepancies in these results. The cross section of the STMT method shows a very broad resonance feature with a maximum $9 \mathrm{eV}$ above threshold, in apparent good agreement with the experimental data. The resonance peak of the MS calculation is centered at even higher energy, almost coinciding with our relaxed-core result; its width and height is somewhat larger than that given by the present FCHF calculation. According to our discussion in Sec. IV A, it is certainly not realistic to expect resonance positions at or even above the experimental value when using the unscreened core-hole potential of the frozen-core model. Similar discrepancies between the STMT result and the iterative Schwinger

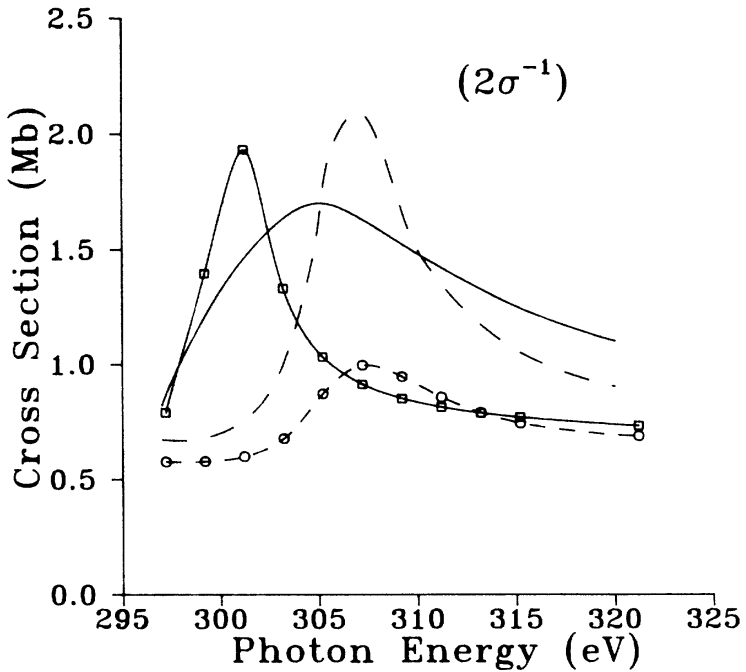

FIG. 2. Photoionization cross sections leading to the $2 \sigma^{-1}$ (C $1 s^{-1}$ ) state of $\mathrm{CO}^{+}, \boxminus \square$, present results (length) in the FCHF approximation; $\theta \theta$, present results (length) in the RCHF approximation; - calculated FCHF results of Ref. 18 using the STMT method; - - - calculated results of Ref. 41 using the MSM method.

static-exchange (FCHF) cross sections have been found for the $K$-shell ionization of $\mathrm{N}_{2}{ }^{20}$

Figure 3 shows the decomposition of the total $\mathrm{C} 1 \mathrm{~s}$ photoionization cross section into the contributions of the $k \sigma$ and $k \pi$ subchannels. To investigate the role of the conjugate contributions we have also plotted (dashed line) the "direct" $k \sigma$ and $k \pi$ cross sections obtained by omitting the conjugate (overlap) part in the amplitude (90b). Note that the overall spectroscopic factor $\left(\left|x_{1 s}\right|^{2}=0.79\right)$ is not taken into account in Fig. 3. In both

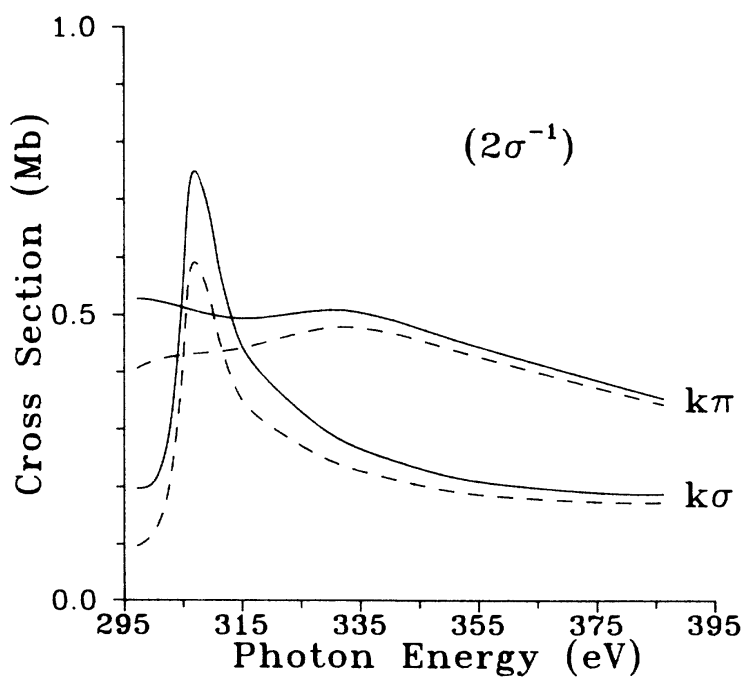

FIG. 3. Partial channel photoionization cross sections leading to the $2 \sigma^{-1}$. (C $1 s^{-1}$ ) state of $\mathrm{CO}^{+}$in the RCHF approximation. - direct plus conjugate cross sections as per Eq. (90b); - - - only direct cross sections using the first term of Eq. (90b). Note that these cross sections omit the overall spectroscopic factor. 
channels we see a significant enhancement of the cross section upon inclusion of the conjugate contribution. The modification due to the bound-free overlap terms is largest at low photoelectron energy and becomes small towards larger energies, as expected. At the resonance position in the $k \sigma$ channel the enhancement of the direct part amounts to $0.16 \mathrm{Mb}$, that is, almost $30 \%$ of the direct value. In the $k \pi$ channel the largest difference between the direct and full cross section is found at threshold, being here about $30 \%$.

A more detailed view of the bound-free overlaps is given in Fig. 4. Here we have plotted the quantities

$$
S_{r}(k)=\frac{8 \pi^{2}}{3 c} \omega k \sum_{l, m}\left|\left\langle\psi_{k l m}^{(-)} \mid \phi_{r}\right\rangle\right|^{2}
$$

as a function of energy for the occupied (frozen) valence orbitals $r=3 \sigma, 4 \sigma, 5 \sigma$, and $1 \pi$. Note that these quantities are dimensionless and when multiplied by the square of the appropriate bound-bound transition matrix element, correspond to a "conjugate transition cross section." It is also important to note that each $l$ component of the conjugate amplitude may interfere with the direct part of the amplitude [see Fig. 90(b)]. In addition, in CO there may be additional interference in the sum over orbital contributions, $\left(r, r^{\prime}\right)$, in the conjugate amplitude.

In the $k \pi$ channel the squared overlap $S_{1 \pi}(k)$ assumes a value of $\geq 2.75$ at threshold and decreases rapidly with increasing photoelectron energy. In the $k \sigma$ curves this type of behavior is superimposed on a resonance enhancement, leading to local maxima (and minima) in the $3 \sigma$ and $4 \sigma$ overlap curves and a distinct shoulder in the $5 \sigma$ curve. The value at threshold is largest for the outermost valence orbital $5 \sigma\left[S_{5 \sigma}(0) \geq 3.5\right]$; distinctly lower values, namely, $S_{4 \sigma}(0) \approx 0.6$ and $S_{3 \sigma}(0) \approx 0.1$, respectively, are found for the inner-valence orbitals.

The squared overlap $S_{r}(k)$ for the core orbitals $\mathrm{O} 1 \mathrm{~s}$ $(1 \sigma)$ and $\mathrm{C} 1 s(2 \sigma)$ are smaller than $10^{-4}$ and 0.03 , respectively. In the strict core-valence separation approxi-

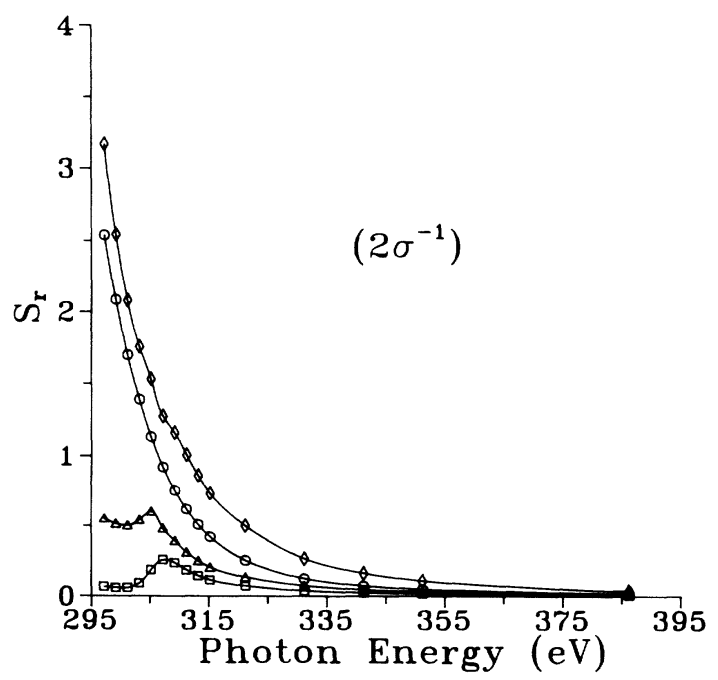

FIG. 4. Frozen bound-relaxed continuum orbital overlaps, $S_{r}$ of Eq. (95). $\boxminus, r=3 \sigma ; \triangle, r=4 \sigma ; \diamond, r=5 \sigma ;-$, $r=1 \pi$. See Sec. VI for more details. mation the frozen- and relaxed-core orbitals are identical and orthogonal to the (relaxed) continuum orbital. However, since this approximation has not been enforced in the calculation of the relaxed orbitals the frozen $1 \mathrm{~s}$ relaxed-continuum overlap integrals are not identically zero. These deviations give an estimate of the error introduced by the core-valence separation approximation.

Figure 5 shows the photoelectron asymmetry parameter $\beta$ calculated in the frozen- and relaxed-core models. The shifting and broadening of the shape resonance by the screening of the $\mathrm{C} 1 s$ hole are apparent in these results. Both the frozen and the relaxed $\beta$ curves have a distinct minimum in the energy regions of the respective frozen and relaxed resonance positions, as seen in the cross sections. Beside the shift in the position, the relaxed curve assumes a somewhat high $\beta$ value at the minimum and is flatter than the frozen one. The experimental data of Truesdale et al. ${ }^{6}$ seem to be in better agreement with the relaxed curve, in particular, if shifted by $2-3 \mathrm{eV}$ to lower energy in order to agree with the experimental resonance position.

Figure 5 also displays the direct part of the relaxed $\beta$ curve obtained by neglecting the conjugate contributions in amplitude (90b). The direct curve differs only little from the solid curve; in particular, above the minimum they are almost identical. This shows that the $\beta$ parameter is little affected by the conjugate contributions.

The $1 s$ photoelectron asymmetry parameters for $\mathrm{CO}$ have been calculated previously by Dill et al. ${ }^{42}$ and by Grimm $^{43}$ using the multiple-scattering model (MSM). These calculations were based on the transition state self-consistent-field (SCF) treatment in which one-half of an electron was removed from the $K$-shell. Correspondingly, only half of the $1 s$-hole screening was accounted for by the scattering potential. The results of such a

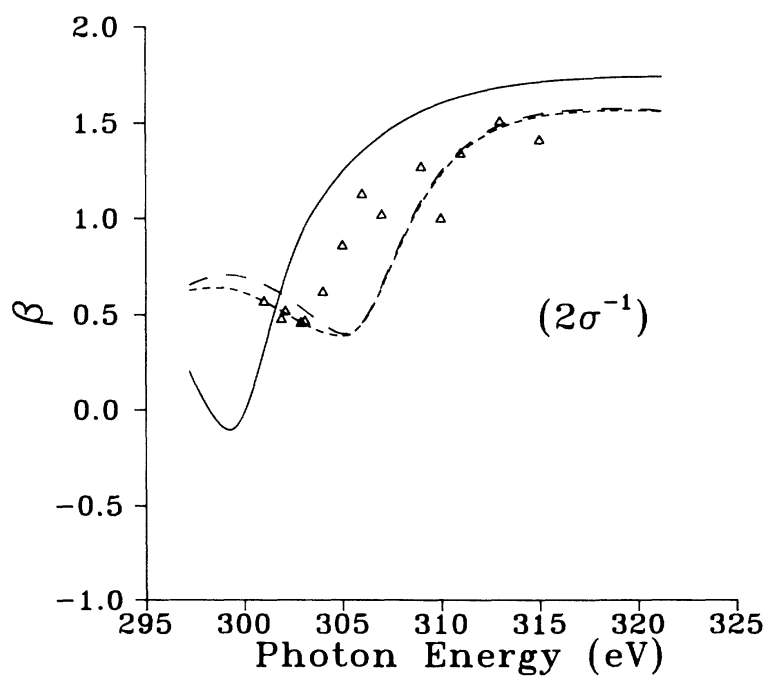

FIG. 5. Photoelectron asymmetry parameters for photoionization leading to the $2 \sigma^{-1}\left(\mathrm{C}_{1 s^{-1}}\right)$ state of $\mathrm{CO}^{+}$. present results (length) in the FCHF approximation; - - present results (length) in the RCHF approximation; --.-, present results (length) only using the direct part of the transition amplitude; $\triangle$, experimental results of Ref. 6 . 
"half-relaxed" method lie, of course, somewhere between those of the frozen- and the fully relaxed-core approximations. In spite of the usually better agreement with experiment there is little physical justification for this procedure, since the shortcoming of the RCHF model is not "overscreening" but neglect of target polarization.

\section{B. $\mathbf{O}(1 s)$ ionization}

The $\mathrm{O} 1 \mathrm{~s}$ photoionization cross sections calculated both in the frozen- and relaxed-core approximations are shown in Fig. 6, along with experimental results of Barrus et al. ${ }^{11}$ (photoabsorption) and Truesdale et al. ${ }^{6}$ and previous theoretical results of Padial et al. ${ }^{18}$ obtained using the FCHF model and STMT methods. As in the $\mathrm{C} 1 s$ cross section, the shape resonance is drastically shifted and broadened in the relaxed potential compared to the frozen-core potential. However, here the differences between the frozen and relaxed calculations are more dramatic. The shift of the resonance position induced by the screening of the $\mathrm{O} 1 s$ hole in the RCHF model is about $10.5 \mathrm{eV}$, which is over $4 \mathrm{eV}$ larger than in the $\mathrm{C} 1 \mathrm{~s}$ cross section; concommitantly the relaxed $\mathrm{O} 1 \mathrm{~s}$ resonance peak is even flatter and more extended than its C $1 s$ counterpart. The difference in the screening effect for the $\mathrm{C} 1 s$ and the $\mathrm{O} 1 s$ hole is, of course, a consequence of the larger electronic reorganization (relaxation) in the presence of a $K$-shell hole at the atom with higher nuclear charge. The relaxation shifts for the $\mathrm{C} 1 \mathrm{~s}$ and $\mathrm{O} 1 \mathrm{~s}$ ionization energies of about 13 and $21 \mathrm{eV}$, respectively, may serve as a measure of this difference.

As in the $\mathrm{C} 1 s$ case, the experimental shape resonance result lies between the frozen and relaxed theoretical curves. Again, we expect that inclusion of polarization in the RCHF potential would lead to better agreement with experiment. However, here the experimental resonance

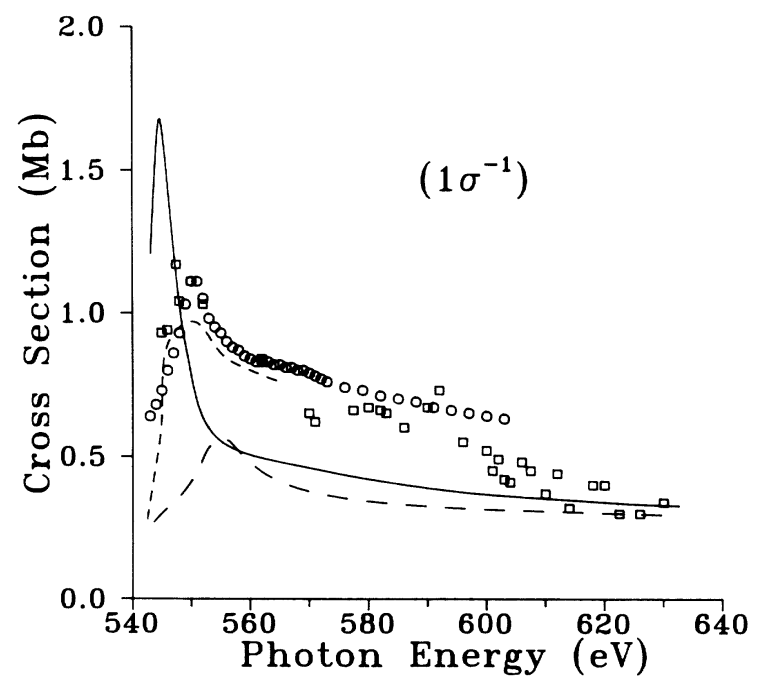

FIG. 6. Photoionization cross sections leading to the $1 \sigma^{-1}$ $\left(\mathrm{O} 1 s^{-1}\right)$ state of $\mathrm{CO}^{+}$. FCHF approximation; - - - present results (length) in the RCHF approximation; ----, calculated FCHF results of Ref. 18 using the STMT method; $O$, experimental results (photoabsorption) of Ref. 11; $\square$, experimental results of Ref. 6. position (about $7.5 \mathrm{eV}$ above threshold) differs by $5.5 \mathrm{eV}$ from the result of the relaxed-core calculation, and this discrepancy appears somewhat too large to be accounted for only by polarization. The overall spectroscopic factor $\left|x_{1 \sigma}\right|^{2}$ contained in the relaxed-core result (but absent in the frozen-core result) was calculated to be 0.74 , a value larger than the previous $a b$ initio result ${ }^{40}$ of 0.61 . The $\mathrm{O}$ $1 s$ cross section calculated by Padial et al. ${ }^{18}$ in the FCHF approximation using the STMT method is again at variance with our frozen-core result even at high photon energy.

The decomposition of the relaxed-core $\mathrm{O} 1 \mathrm{~s}$ cross section into the $k \sigma$ and $k \pi$ subchannels is shown in Fig. 7. Also plotted here are the direct parts of the respective cross sections, thus making apparent the role of the conjugate contributions. As in the $\mathrm{C} 1 s$ ionization they result in a substantial enhancement of the cross section in both channels. Remarkably, the direct $k \pi$ cross section increases even at $100 \mathrm{eV}$ above threshold, and the enhancement by the conjugate contribution is still of the order of $10 \%$.

The frozen and relaxed photoelectron asymmetry parameters for $\mathrm{O} 1 s$ ionization are plotted in Fig. 8. For both curves the $\beta$ values at low energies are higher than for $\mathrm{C} 1 s$ ionization. As in the cross sections, the differences between FCHF and RCHF $\beta$ 's are qualitatively the same as in $C(1 s)$ photoionization. The relaxed $\beta$ curve exhibits a pronounced maximum near threshold. The synchrotron data by Truesdale et $a l .{ }^{6}$ are somewhat scarce and scattered, making the comparison between theory and experiment inconclusive.

\section{SUMMARY AND CONCLUSIONS}

The relaxed-core Hartree-Fock model used here to study molecular $K$-shell photoionization cross sections and angular distributions contains the following approximations.

(i) The $N$-electron initial state is given by its $\mathrm{HF}$ repre-

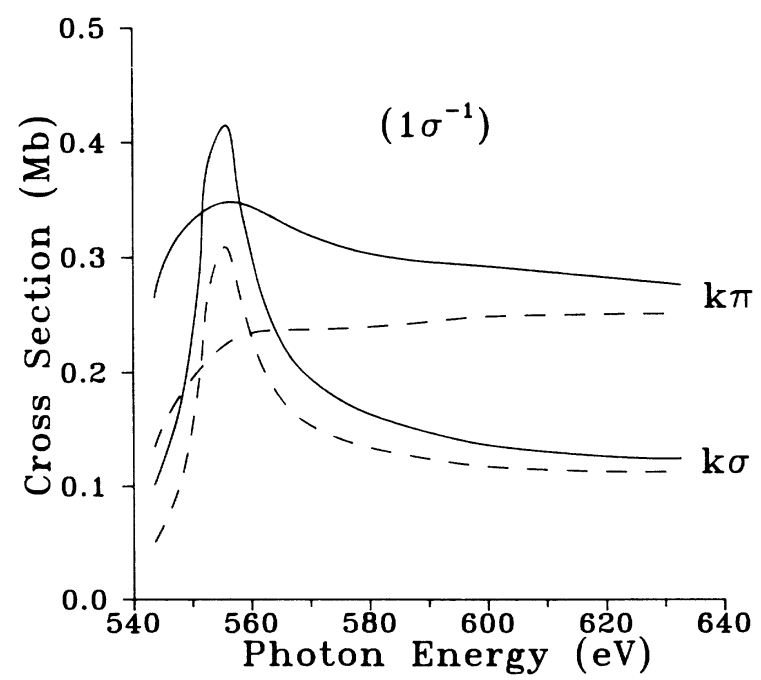

FIG. 7. Same as Fig. 3 except for photoionization leading to the $1 \sigma^{-1}\left(\mathrm{O}^{1 s^{-1}}\right)$ state of $\mathrm{CO}^{+}$. 
sentation, that is, as a Slater determinant of "frozen" orbitals.

(ii) The relaxed orbitals generated by a HF calculation for the $1 s$-hole state are used to represent the ion and the potential for the electron-ion scattering. The photoelectron continuum orbitals are constrained to be orthogonal to the occupied relaxed orbitals.

(iii) The $N$-electron final state is - apart from spin (and spatial symmetry) coupling-represented by an antisymmetrized product of the ionic state and the continuum orbital.

In addition, we have introduced the core-valence separation approximation, which is justified in the case of $K$ shell ionization due to the large energy separation between the $1 s$ and valence levels and to the small coupling matrix elements for states with differing $K$-shell occupations. In the CVS approximation the $N$-electron initial and final states are strictly orthogonal and thus one gets rid of unphysical contributions to the RCHF $N$-electron transition moment. Furthermore, in the CVS approximation the familiar IVO form of the static-exchange potential is consistent with the general variational principle used to derive the RCHF potential.

Compared to the more widely used frozen-core Hartree-Fock calculations, the essential computational complication of the RCHF model arises from the nonorthogonality of the frozen and relaxed orbitals in the initial and final states, respectively. The method proposed here to evaluate $N$-electron matrix elements for determinants with mutually nonorthogonal orbitals has proven to be particularly practical and transparent in the case of the RCHF transition moment. Besides the computational aspect, the new formulation also leads to a conceptual clarification, since it allows one to make a distinction between the direct and the conjugate parts of the transition moment. The latter part arises due to the lack of orthogonality between the (relaxed) continuum orbital

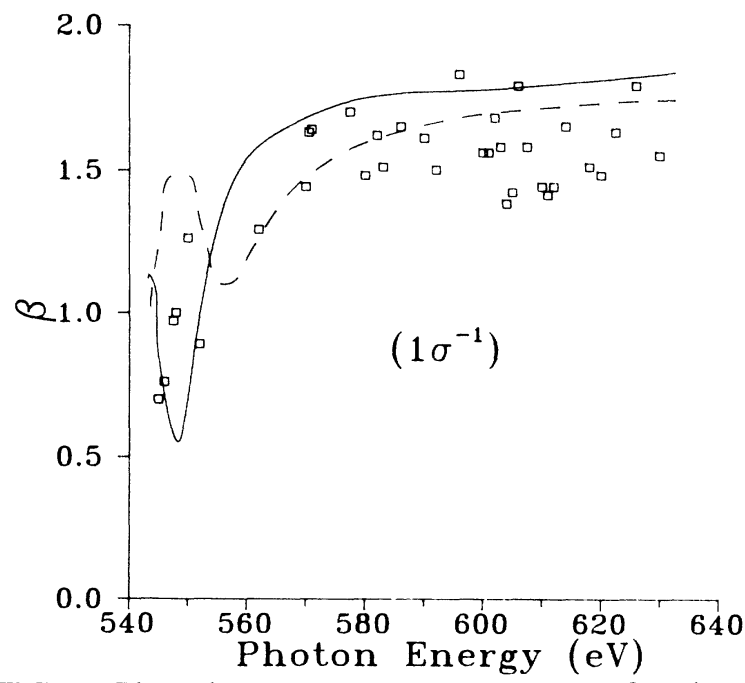

FIG. 8. Photoelectron asymmetry parameters for photoionization leading to the $1 \sigma^{-1}\left(\mathrm{O} 1 s^{-1}\right)$ state of $\mathrm{CO}^{+}$. present results (length) in the FCHF approximation; - - present results (length) in the RCHF approximation; $\square$, experimental results of Ref. 6 . and the frozen occupied orbitals, which is reflected in the bound-free overlap integrals introduced here. The direct part, on the other hand, is characterized by the occurrence of the usual bound-free dipole (transition) integrals, and prevails, of course, also in the case of orthogonality between bound and continuum orbitals. The effect of the conjugate part can be viewed as a modification of the bound-free dipole integral, essentially projecting out the occupied frozen orbital contributions in the continuum orbital. An explicit study of the relative importance of these contributions was made for the case of $\mathrm{C} 1 s$ ionization. Here the conjugate contributions were seen to enhance quite substantially the "direct" cross section at low photoelectron energy. Towards higher energy their influence becomes small, as the bound-free overlap integrals tend to vanish rapidly.

To better understand the potential and the limitation of the RCHF approximation we have analyzed its results by means of perturbation theory. With respect to energies, i.e., resonance positions or term values (below the $1 s$ threshold) the RCHF description is complete through first order but only partially recovers the correct secondorder contributions. As expected, the RCHF comprises, apart from exchange terms, contributions associated with ionic relaxation. In particular, the screening of the $1 s$ hole by the reorganization of the valence electrons is correctly described in second order. It is this effect that leads to considerably larger resonance energies than calculated using the unscreened $1 s$-hole potential of the FCHF model. The analysis in second order reveals also the main deficiency of the RCHF approximation: the neglect of target polarization, i.e., effects associated with the response of the ion to the presence of the photoelectron. The polarization correction makes the ion-electron potential more attractive and leads to a lowering of resonance positions. The discrepancies found between the RCHF results and experimental results have to be attributed mainly to this source. The analysis by perturbation theory has also been applied to the $N$-electron transition moment. Apart from contributions vanishing in the CVS approximation, here a consistent first-order treatment is achieved at the RCHF level.

The picture emerging from this study shows that, recognizing its limitations, the $\mathrm{RCHF}$ approximation establishes a good approach to the theoretical description of $K$-shell ionization, and is, indeed, superior to the FCHF model which ignores the important relaxation effects. A very efficient computational scheme, combining our methods for solving the single-particle scattering equations with the new formulation of the transition moment, is now available for further such studies.

\section{ACKNOWLEDGMENTS}

This material is based upon research supported by the National Science Foundation under Grant No. CHE8521391. One of us (J.S.) wishes to express his gratitude for the hospitality given during his stay at Caltech's Laboratory of Chemical Physics. He also acknowledges funding by the German Federal Ministry for Research and Technology under the Contract No. 05 352AX-3. The authors 
acknowledge use of the resources of the San Diego Supercomputer Center which is supported by the National Science Foundation.

\section{APPENDIX: OVERLAP AND TRANSITION MATRIX ELEMENTS FOR FROZEN AND RELAXED STATES}

In the following we consider two distinct sets $\left\{\left|\phi_{i}\right\rangle\right\}$ and $\left\{\left|\psi_{i}\right\rangle\right\}$ of orbitals, e.g., the sets of frozen and relaxed HF orbitals discussed in Sec. II. The associated operators in second-quantized notation are denoted by $a_{i}$ and $c_{i}$, respectively. The Slater determinants of the first $N$ orbitals,

$$
\begin{aligned}
& \left|\Phi_{0}^{N}\right\rangle=\left|\phi_{1} \cdots \phi_{N}\right|, \\
& \left|\overline{\boldsymbol{\Phi}}_{0}^{N}\right\rangle=\left|\psi_{1} \cdots \psi_{N}\right|,
\end{aligned}
$$

within each set are referred to as the frozen and relaxed ground states, respectively. Further definitions needed are the relaxed-frozen overlap matrix $\underline{S}$,

$$
S_{i j}=\left\langle\psi_{i} \mid \phi_{j}\right\rangle, \quad i, j \leq N
$$

and the matrix $\underline{d}$ of dipole integrals

$$
d_{i j}=\left\langle\psi_{i}|\hat{d}| \phi_{j}\right\rangle, \quad i, j \leq N .
$$

Both matrices are $N \times N$ matrices defined for the sets of occupied orbitals $1, \ldots, N$. In a more compact (matrix) notation we may write

$$
\begin{aligned}
& \underline{S}=\left(\boldsymbol{\psi}^{\dagger}, \boldsymbol{\phi}\right), \\
& \underline{d}=\left(\boldsymbol{\psi}^{\dagger}, \hat{d} \phi\right),
\end{aligned}
$$

where $\phi=\left(\left|\phi_{1}\right\rangle, \ldots,\left|\phi_{N}\right\rangle\right)$ and $\psi=\left(\left|\psi_{1}\right\rangle, \ldots,\left|\psi_{N}\right\rangle\right)$ denote rows of the first $N$ frozen and relaxed orbitals, respectively. The biorthogonalization procedure for the two sets $\phi$ and $\psi$ of mutually nonorthogonal functions consists of determining the unitary transformations

$$
\begin{aligned}
& \widetilde{\phi}=\phi \underline{U}, \\
& \widetilde{\psi}=\psi \underline{V}
\end{aligned}
$$

within each of these sets, fulfilling the condition that the overlap matrix for the transformed orbitals be diagonal $(\underline{\Delta}):$

$$
\left(\widetilde{\boldsymbol{\psi}}^{\dagger}, \widetilde{\boldsymbol{\phi}}\right)=\underline{V}^{\dagger}\left(\boldsymbol{\psi}^{\dagger}, \boldsymbol{\phi}\right) \underline{U}=\underline{V}^{\dagger} \underline{\underline{S}} \underline{U}=\underline{\Delta} .
$$

As in (A1) and (A2) one may define Slater determinants $\left|\widetilde{\Phi}_{0}^{N}\right\rangle$ and $\left|\widetilde{\Phi}_{0}^{N}\right\rangle$ for the sets of biorthogonalized orbitals $\widetilde{\phi}$ and $\widetilde{\psi}$. Since $\underline{U}$ and $\underline{V}$ are unitary matrices the relations

$$
\left|\widetilde{\Phi}_{0}^{N}\right\rangle=\operatorname{det}(\underline{U})\left|\Phi_{0}^{N}\right\rangle
$$

$$
\left|\widetilde{\boldsymbol{\Phi}}_{0}^{N}\right\rangle=\operatorname{det}(\underline{\boldsymbol{V}})\left|\overline{\boldsymbol{\Phi}}_{0}^{N}\right\rangle
$$

hold, where

$$
|\operatorname{det}(\underline{U})|=|\operatorname{det}(\underline{V})|=1 .
$$

Using these relations and Eq. (A6) one readily arrives at the well-known result

$$
\left\langle\bar{\Phi}_{0}^{N} \mid \Phi_{0}^{N}\right\rangle=\operatorname{det}(\underline{S})
$$

for the relaxed-frozen ground-state overlap, which, of course, can also be obtained directly. Next we consider the $(N-1)$-electron overlap integral of the relaxed and frozen single-hole states $c_{q}\left|\bar{\Phi}_{0}^{N}\right\rangle$ and $a_{p}\left|\Phi_{0}^{N}\right\rangle$, respectively. Using the biorthogonalized orbitals we may write

$$
\begin{aligned}
& \left\langle\bar{\Phi}_{0}^{N}\left|c_{q}^{\dagger} a_{p}\right| \Phi_{0}^{N}\right\rangle \\
& \quad=\sum_{r, s} V_{q r}^{*} U_{p s}\left\langle\widetilde{\Phi}_{0}^{N}\left|\widetilde{c}_{r}^{\dagger} \widetilde{a}_{s}\right| \widetilde{\Phi}_{0}^{N}\right\rangle \operatorname{det}\left(\underline{U} \underline{V}^{*}\right)^{-1} .
\end{aligned}
$$

Here the operators $\widetilde{c}_{r}$ and $\widetilde{a}_{s}$ are defined with respect to the biorthogonalized orbitals with transformation relations reading

$$
\begin{aligned}
& a_{p}=\sum_{s=1}^{N} U_{p s} \widetilde{a}_{s}, \\
& c_{q}=\sum_{r=1}^{N} V_{q r} \widetilde{r}_{r} .
\end{aligned}
$$

Now the matrix element on the right-hand side of Eq. (A9) is readily evaluated, yielding

$$
\left\langle\widetilde{\Phi}_{0}^{N}\left|\widetilde{c}_{r}^{\dagger} \widetilde{a}_{s}\right| \bar{\Phi}_{0}^{N}\right\rangle=\delta_{r s} \frac{\left\langle\widetilde{\Phi}_{0}^{N} \mid \widetilde{\Phi}_{0}^{N}\right\rangle}{\left\langle\widetilde{\psi}_{r} \mid \widetilde{\phi}_{r}\right\rangle} .
$$

Inserting this result in Eq. (A9) we obtain

$$
\left\langle\bar{\Phi}_{0}^{N}\left|c_{q}^{\dagger} a_{p}\right| \Phi_{0}^{N}\right\rangle=\sum_{r} V_{q r}^{*} U_{p r}\left(\left\langle\widetilde{\psi}_{r} \mid \widetilde{\phi}_{r}\right\rangle\right)^{-1}\left\langle\bar{\Phi}_{0}^{N} \mid \Phi_{0}^{N}\right\rangle .
$$

Comparison of the expression on the right-hand side with the relation

$$
\underline{S}^{-1}=\underline{U} \underline{\Delta}^{-1} \underline{V}^{\dagger},
$$

which follows from Eq. (A6), leads to the final result

$$
\left\langle\widetilde{\Phi}_{0}^{N}\left|c_{q}^{\dagger} a_{p}\right| \Phi_{0}^{N}\right\rangle=\left(\underline{S}^{-1}\right)_{p q}\left\langle\bar{\Phi}_{0}^{N} \mid \Phi_{0}^{N}\right\rangle .
$$

The important feature of this result is that all "biorthogonalized" quantities have dropped out and the final expression requires only the simple overlap matrix $\underline{S}$. Proceeding in the same way we may evaluate the transition moment for the single-hole states:

$$
\begin{aligned}
& \left\langle\bar{\Phi}_{0}^{N}\left|c_{q}^{\dagger} \hat{D} a_{p}\right| \Phi_{0}^{N}\right\rangle=\sum_{r, s} V_{q r}^{*} U_{p s}\left\langle\tilde{\Phi}_{0}^{N}\left|\tilde{c}_{r}^{\dagger} \hat{D} \widetilde{a}_{s}\right| \widetilde{\Phi}_{0}^{N}\right\rangle \operatorname{det}\left(\underline{U} \underline{V}^{*}\right)^{-1} \\
& =\left[\sum_{\substack{r, s \\
r \neq s}} V_{q r}^{*} U_{p s} \frac{-\left\langle\tilde{\psi}_{s}|\hat{d}| \widetilde{\phi}_{r}\right\rangle}{\left\langle\widetilde{\psi}_{s} \mid \widetilde{\phi}_{s}\right\rangle\left\langle\widetilde{\psi}_{r} \mid \widetilde{\phi}_{r}\right\rangle}+\sum_{r} V_{q r}^{*} U_{p r}\left(\sum_{\substack{i \\
i \neq r}} \frac{\left\langle\widetilde{\psi}_{i}\left|\hat{d}_{i}\right| \tilde{\phi}_{i}\right\rangle}{\left\langle\tilde{\phi}_{i}\right\rangle\left\langle\widetilde{\psi}_{r} \mid \widetilde{\phi}_{r}\right\rangle}\right]\right]\left\langle\bar{\Phi}_{0}^{N} \mid \Phi_{0}^{N}\right\rangle
\end{aligned}
$$

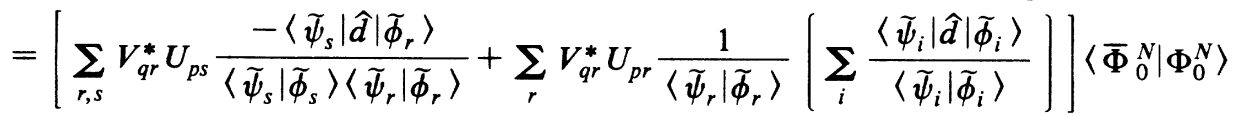

$$
\begin{aligned}
& =\left[-\left(\underline{S}^{-1} \underline{d}^{-1}\right)_{p q}+\left(\underline{S}^{-1}\right)_{p q} \operatorname{Tr}\left(\underline{d} \underline{S}^{-1}\right)\right]\left\langle\bar{\Phi}_{0}^{N} \mid \Phi_{0}^{N}\right\rangle \text {. }
\end{aligned}
$$


Here $\underline{d}$ is the matrix of relaxed-frozen dipole integrals defined by Eq. (A4). For the following quantities we give only the results.

(i) Overlap relaxed single excitation-frozen ground state

$$
\left\langle\bar{\Phi}_{0}^{N}\left|c_{l}^{\dagger} c_{j}\right| \Phi_{0}^{N}\right\rangle=\sum_{r=1}^{N}\left\langle\psi_{j} \mid \phi_{r}\right\rangle\left(\underline{S}^{-1}\right)_{r l}\left\langle\bar{\Phi}_{0}^{N} \mid \Phi_{0}^{N}\right\rangle \text {. }
$$

(ii) Transition moment relaxed single excitation-frozen ground state

$$
\left\langle\bar{\Phi}_{0}^{N}\left|c_{l}^{\dagger} c_{j} \hat{D}\right| \Phi_{0}^{N}\right\rangle=\left(\sum_{r}\left\langle\psi_{j}|d| \phi_{r}\right\rangle\left(\underline{S}^{-1}\right)_{r l}+\Sigma\left\langle\psi_{j} \mid \phi_{r}\right\rangle\left(-\underline{S}^{-1} \underline{d}^{-1}+\operatorname{Tr}\left(\underline{d}^{-1} \underline{S}^{-1}\right)_{r l}\right)\left\langle\bar{\Phi}_{0}^{N} \mid \Phi_{0}^{N}\right\rangle .\right.
$$

(iii) Overlap relaxed $2 h$-1 $p$ satellite-frozen single-hole state $\left\langle\bar{\Phi}_{0}^{N}\left|c_{l}^{\dagger} c_{h}^{\dagger} c_{j} a_{r}\right| \Phi_{0}^{N}\right\rangle$ [see Eq. (27)].

(iv) Transition moment relaxed $2 h-1 p$ satellite-frozen single-hole state $\left\langle\bar{\Phi}_{0}^{N}\left|c_{l}^{\dagger} c_{h}^{\dagger} c_{j} \hat{D} a_{r}\right| \Phi_{0}^{N}\right\rangle$ [see Eq. (28)].

*Permanent address: Physikalisch-Chemisches Institut, Universität Heidelberg, D-6900 Heidelberg, Federal Republic of Germany.

${ }^{1}$ K. Siegbahn, C. Nordling, C. Johansson, J. Hedman, P. F. Heden, K. Hamrin, U. Gelius, T. Bergmark, L. O. Werme, R. Manne, and Y. Baer, ESCA Applied to Free Molecules (North-Holland, Amsterdam, 1971).

${ }^{2}$ H. Siegbahn and L. Karlsson, in Handbuch der Physik, edited by W. Mehlhorn (Springer, Berlin, 1982), Vol. 31.

${ }^{3}$ U. Gelius, J. Electron Spectrosc. Relat. Phenomn. 5, 985 (1974).

${ }^{4}$ V. McKoy, T. A. Carlson, and R. R. Lucchese, J. Phys. Chem. 88, 3188 (1984).

${ }^{5}$ P. H. Kobrin, S. Southworth, C. M. Truesdale, D. W. Lindle, U. Becker, and D. A. Shirley, Phys. Rev. A 29, 194 (1984).

${ }^{6}$ C. M. Truesdale, S. H. Southworth, P. H. Kobrin, U. Becker, D. W. Lindle, H. G. Kerkhoff, and D. A. Shirley, Phys. Rev. Lett. 50, 1265 (1983); C. M. Truesdale, D. W. Lindle, P. H. Kobrin, U. Becker, H. G. Kerkhoff, P. A. Heimann, T. A. Ferrett, and D. A. Shirley, Phys. 80, 2319 (1984).

${ }^{7}$ D. W. Lindle, C. M. Truesdale, P. H. Kobrin, T. A. Ferrett, P. A. Heimann, U. Becker, H. G. Kerkhoff, and D. A. Shirley, J. Chem. Phys. 81, 5375 (1984).

${ }^{8}$ See, e.g., C. E. Brion, S. Daniel, R. Sodlei, and A. P. Hitchcock, in X-Ray and Atomic Inner-Shell Physics, edited by B. Craseman (AIP, New York, 1982), and references therein.

${ }^{9}$ A. S. Vinogradov, B. Shlarbaum, and T. M. Zimkina, Opt. Spectrosc. (USSR) 36, 383 (1974).

${ }^{10}$ A. Bianconi, H. Petersen, F. C. Brown, and R. Z. Bachrach, Phys. Rev. A 17, 1907 (1978).

${ }^{11}$ D. M. Barrus, R. L. Blake, A. J. Burek, K. C. Chambers, and A. L. Pregenzer, Phys. Rev. A 20, 1045 (1979).

${ }^{12}$ A. Reimer, J. Schirmer, J. Feldhaus, A. M. Bradhsaw, U. Becker, H. G. Kerkhoff, B. Langer, D. Szostak, R. Wehlitz, and W. Braun, Phys. Rev. Lett. 57, 1707 (1986).

${ }^{13}$ D. Dill and J. L. Dehmer, J. Chem. Phys. 61, 691 (1974).

${ }^{14} \mathrm{P}$. W. Langhoff, in Electron-Molecule and Photon-Molecule Collisions, edited by T. Rescigno, V. McKoy, and B. Schneider (Plenum, New York, 1979).

${ }^{15}$ R. R. Lucchese, K. Takatsuka, and V. McKoy, Physics Rep. 131, 147 (1986).

${ }^{16}$ J. L. Dehmer and D. Dill, J. Chem. Phys. 65, 5327 (1976).

${ }^{17}$ T. N. Rescigno and P. W. Langhoff, Chem. Phys. Lett. 51, 65 (1977).
${ }^{18}$ N. Padial, G. Csanak, V. McKoy, and P. W. Langhoff, J. Chem. Phys. 69, 92992 (1978).

${ }^{19}$ R. R. Lucchese and V. McKoy, Phys. Rev. A 26, 1406 (1982).

${ }^{20}$ D. L. Lynch and V. McKoy, Phys. Rev. A 30, 1561 (1984).

${ }^{21}$ R. L. Martin and D. A. Shirley, J. Chem. Phys. 64, 3685 (1976).

22J. Berkowitz, J. L. Dehmer, Y. K. Kim, and J. P. Descleaux, J. Chem. Phys. 61, 2556 (1974).

${ }^{23}$ T. Åberg, Phys. Rev. 156, 35 (1967).

${ }^{24}$ A. F. Starace, in Ref. 2.

${ }^{25}$ T. Ishihara, J. Mizuno, and T. Watanabe, Phys. Rev. A 22, 1552 (1980).

${ }^{26}$ J. Schirmer, M. Braunstein, and V. McKoy, J. Chem. Phys. (to be published).

${ }^{27}$ R. Arneberg, J. Müller, and R. Manne, Chem. Phys. 64, 249 (1982).

${ }^{28}$ I. Cacelli, V. Carravetta, and R. Moccia, J. Phys. B 16, 1895 (1983).

${ }^{29}$ W. von Niessen, J. Schirmer, and L. S. Cederbaum, Comput. Phys. Rep. 1, 59 (1984).

${ }^{30}$ L. S. Cederbaum, W. Domcke, and J. Schirmer, Phys. Rev. A 82, 206 (1980).

${ }^{31}$ For example, see B. H. Bransden, Atomic Collision Theory (Benjamin, New York, 1970).

${ }^{32}$ W. J. Hunt and W. Goddard, Chem. Phys. Lett. 3, 414 (1969).

${ }^{33}$ J. Schirmer, Phys. Rev. A 26, 2395 (1982).

${ }^{34}$ J. Schirmer, O. Walter, and L. S. Cederbaum, Phys. Rev. A 28, 1237 (1983).

${ }^{35} \mathrm{See}$, for example, A. L. Fetter and J. D. Walecka, Quantum Theory of Many-Particle Systems (McGraw-Hill, New York, 1971).

${ }^{36}$ Gaussian Basis Sets for Molecular Calculations, edited by S. Huzinaga (Elsevier, Amsterdam, 1984).

${ }^{37}$ R. B. Lucchese and V. McKoy, Phys. Rev. A 21, 112 (1980).

${ }^{38}$ A. R. Edmonds, Angular Momentum in Quantum Mechanics (Princeton University, Princeton, 1960).

${ }^{39}$ R. B. Kay, Ph. E. Van der Leeuw, and M. J. Van der Wiel, J. Phys. B 10, 2513 (1977).

${ }^{40}$ G. Angonoa, O. Walter, and J. Schirmer, J. Chem. Phys. 87, 6789 (1987).

${ }^{41}$ J. L. Dehmer and D. Dill, Argonne National Laboratory Report No. ANL-77-65, 1977 (unpublished), p. 65.

${ }^{42}$ D. Dill, S. Wallace, J. Siegel, and J. L. Dehmer, Phys. Rev. Lett. 42, 411 (1978).

${ }^{43}$ F. A. Grimm, Chem. Phys. 53, 71 (1980). 